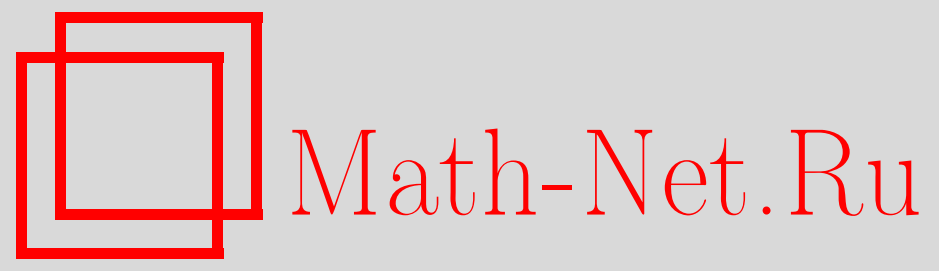

Нгуен Ван Хоа, О замкнутых классах $k$-значной логики, самодвойственных относительно транзитивных групп, Дискрет. матем., 1996, том 8, выпуск 1, 129-156

DOI: https://doi.org/10.4213/dm513

Использование Общероссийского математического портала Math-Net.Ru подразумевает, что вы прочитали и согласны с пользовательским соглашением http://www.mathnet.ru/rus/agreement

Параметры загрузки:

IP : 34.227 .88 .159

26 апреля 2023 г., $11: 18: 19$

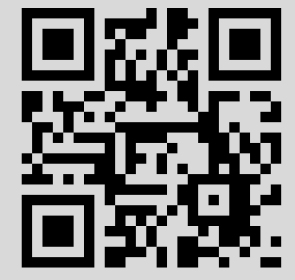




\title{
О замкнутых классах $k$-значной логики, самодвойственных относительно транзитивных групп
}

\author{
(C) 1996 г. Нгуен Ван Хоа
}

\begin{abstract}
Доказано, что при $m \geqslant[k / 2]+1$ структура замкнутых классов $k$-значной логики, самодвойственных относительно $m$-транзитивных подгрупп симметрических групп, конечна и состоит из замкнутых классов, имеющих конечный базис.
\end{abstract}

\section{1. Введение}

Известно, что решетка замкнутых классов (з.к.) $k$-значной логики счетна и имеет эффективное описание при $k=2$ [1]. Однако при $k \geqslant 3$ она континуальна [2]. По этой причине естественный интерес представляет исследование структур з.к. $k$-значной логики при отождествлении похожих в определенном смысле функций. Похожесть функций может играть роль многозначного оператора над функциями, который вместе с операцией суперпозиции образует новый более сильный оператор замыкания.

В данной работе похожесть функций определяется с помощью понятия двойственности функции. Пусть $S_{k}$ - симметрическая группа степени $k, G$ - подгруппа группы $S_{k}$. Пусть $G^{*}$ есть такое бинарное отношение на $P_{k}$, что $f G^{*} g$ тогда и только тогда, когда $s^{-1} f\left(s\left(x_{1}\right), \ldots, s\left(x_{n}\right)\right)=g\left(x_{1}, \ldots, x_{n}\right)$ для некоторого $s \in G$. Очевидно, что $G^{*}$ является отношением эквивалентности на $P_{k}$.

$G^{*}$-замыкание $[\Sigma]_{G^{*}}$ для любой системы функций $\Sigma \subseteq P_{k}$ индуктивно определяется следующими правилами:

(1) всякая функция из $\Sigma$ принадлежит $[\Sigma]_{G^{*}}$;

(2) если $f \in[\Sigma]_{G^{*}}$ и $f G^{*} g$, то $g \in[\Sigma]_{G^{*}}$;

(3) если $M \subseteq[\Sigma]_{G^{*}}$, то $[M] \subseteq[\Sigma]_{G^{*}}$.

Функции рассматриваются с точностью до фиктивных переменных. Множество $M$ функций из $P_{k}$ называется $G^{*}$-замкнутым классом, если $[M]_{G^{*}}=M$. Множество функций $\Sigma \subset M \subset P_{k}$ называется $G^{*}$-полным в $M$, если $[\Sigma]_{G^{*}}=$ $M$. Естественным образом вводятся понятия $G^{*}$-критериальной системы, $G^{*}$ предполного класса, $G^{*}$-базиса, $G^{*}$-порядка. 
Очевидно, что $G^{*}$-з. к. является одновременно з.к. в обычном смысле. Поэтому можно считать структуру $L_{G^{*}}\left(P_{k}\right)$ всех $G^{*}$-з. к. некоторой подструктурой первоначальной структуры з.к., которая состоит из всех з.к. типа $G^{*}$. Такую подструктуру будем называть $G^{*}$-подструктурой структуры $L\left(P_{k}\right)$ з.к. $k$-значной логики. При единичной подгруппе $\left(G^{*}=e_{k}\right)$ имеем $L_{e_{k}}\left(P_{k}\right)=L\left(P_{k}\right)$. Описание структуры $L_{S_{k}^{*}}\left(P_{k}\right)$ имеется в работах $[6,10,14]$. В данной работе будет доказано, что $L_{G^{*}}\left(P_{k}\right)$ конечна и состоит из з. к., имеющих конечный базис, если группа $G m$-транзитивна, где $m \geqslant[k / 2]+1$ ([a] обозначает наибольшее целое число, не большее $a$ ).

\section{2. Основные понятия и обозначения}

Пусть $E_{k}=\{0,1, \ldots, k-1\}, k \geqslant 2, P_{k}^{n}=\left\{f^{n} \mid f^{n}: E_{k}^{n} \rightarrow E_{k}\right\}, n=1,2, \ldots$,

$$
P_{k}=\bigcup_{n \geqslant 1} P_{k}^{n}
$$

Через $S\left(E_{k}\right), A\left(E_{k}\right)\left(S_{k}(M), A_{k}(M)\right.$, где $\left.M \subset E_{k},|M|<k\right)$ обозначим соответственно симметрическую, знакопеременную группы, действующие на $E_{k}($ на $M)$, а через $V\left(E_{4}\right)\left(V_{k}(M),|M|=4<k\right)$ четвертую группу Клейна на $E^{4}$ (на $\left.M\right)$. Как 3. к. функций из $P_{k}\left(P_{4}\right)$ множества $S\left(E_{k}\right), A\left(E_{k}\right)\left(V\left(E_{4}\right)\right)$ имеют соответственно еще обозначения $P S_{k}, P A_{k}\left(P V_{4}\right)$. Единичная перестановка из $S\left(E_{k}\right)$ составляет единичную подгруппу $P_{e_{k}}$. Для любого $t \leqslant k-1$ пусть

$$
s_{t}=\left(\begin{array}{c}
k \\
t+1
\end{array}\right)
$$

а $I_{i}^{t+1}, i=1, \ldots, s_{t}$, есть все $(t+1)$-элементные подмножества множества $E_{k}$, где $I_{1}^{t+1}=\{0,1, \ldots, t\}$. Пусть

$$
\begin{aligned}
& B_{I_{i}^{t}}^{n}=\left\{\left(\alpha_{1}, \ldots, \alpha_{n}\right) \in E_{k}^{n} \mid\left\{\alpha_{1}, \ldots, \alpha_{n}\right\} \subseteq I_{i}^{t}\right\}, \\
& T_{k, t}^{n}=\left\{\left(\alpha_{1}, \ldots, \alpha_{n}\right) \in E_{k}^{n} \mid\left\{\alpha_{1}, \ldots, \alpha_{n}\right\}=t\right\} .
\end{aligned}
$$

$B_{I_{i}^{t}}^{n} f, B_{i} f, \vec{B}_{i}^{f}-$ ограничения функции $f$ на $B_{I_{i}^{t}}^{n}, B_{i}^{n}=B_{I_{i}^{t}}^{n}$ и на множество

$$
\vec{B}_{i}^{n}=\bigcup_{s=1}^{s_{i}-1} B_{I_{s}^{i}}^{n}
$$

соответственно, где $t=2, \ldots, k, i=1, \ldots, s_{t-1}$. Для любого множества функций $M$ из $P_{k}$ пусть

$$
\begin{aligned}
B_{i} M & =\bigcup_{f \in M}\left\{B_{i} f\right\}, \quad \vec{B}_{i} M=\bigcup_{f \in M}\left\{\vec{B}_{i} f\right\}, \quad 2 \leqslant i \leqslant k-1, \\
G^{*}(M) & =\left\{f \in P_{k} \mid f G^{*} g \text { для некоторого } g \in M\right\} .
\end{aligned}
$$

Пусть $\tilde{a}_{j}^{1}=(j, j+1, j+2,3), \tilde{a}_{j}^{2}=(j+1, j, j+2,3), j=0,1,2$ (сложение здесь рассматривается по модулю 3$)$. Пусть

$$
V_{j}^{i}=\bigcup_{\pi \in V\left(E_{4}\right)}\left\{\pi\left(\tilde{a}_{j}^{i}\right)\right\}, \quad j=1,2, \quad j=0,1,2 .
$$


При $k \geqslant 3$ пусть

$$
\begin{aligned}
d(x, y, z) & = \begin{cases}x, & \text { если } x=y, \\
z & \text { в противном случае; }\end{cases} \\
s(x, y, z) & = \begin{cases}z, & \text { если } x=y, \\
y, & \text { если } x=z, \\
x & \text { в остальных случаях; }\end{cases} \\
l_{n}\left(x_{1}, \ldots, x_{n}\right) & = \begin{cases}x_{1}, & \text { если }\left|\left\{x_{1}, \ldots, x_{n}\right\}\right|=n, \\
x_{n} & \text { в противном случае; }\end{cases} \\
f^{\overrightarrow{0}}(x, y) & = \begin{cases}x & \text { на }\{0,1\}^{2}, \\
y & \text { на остальных наборах. }\end{cases}
\end{aligned}
$$

При $k=4$ пусть

$$
\begin{aligned}
f^{\overrightarrow{0}}(x, y) & = \begin{cases}x & \text { на }\{0,1\}^{2} \cup\{2,3\}^{2}, \\
y & \text { на остальных наборах }\end{cases} \\
f_{0}(x, y, z) & =x+y+z, \quad \gamma_{4}(x, y)=3 x+2 y
\end{aligned}
$$

(сложение + рассматривается в поле Галуа $G F(4))$.

Для любой перестановки $\pi \in S\left(E_{k}\right)$ и любой функции $f \in P_{k}^{n}$ пусть

$$
f^{\pi}\left(x_{1}, \ldots, x_{n}\right)=\langle\pi\rangle\left(f\left(x_{1}, \ldots, x_{n}\right)\right)=\pi^{-1} f\left(\pi\left(x_{1}\right), \ldots, \pi\left(x_{n}\right)\right),
$$

а для любого набора $\tilde{a}=\left(a_{1}, \ldots, a_{n}\right) \in E_{k}^{n}$ пусть $\pi(\tilde{a})=\left(\pi\left(a_{1}\right), \ldots, \pi\left(a_{n}\right)\right)$, $M_{\tilde{a}}=\left\{a_{1}, \ldots, a_{n}\right\}$. Для данного $G^{*}$-з.к. $K$ через $\operatorname{BAS}(K), \operatorname{ORD}(K), \operatorname{CR}(K)$, $\mathrm{BAS}_{G^{*}}(K), \mathrm{ORD}_{G^{*}}(K), \mathrm{CR}_{G^{*}}(K)$ обозначим базис, порядок, критериальную систему, $G^{*}$-базис, $G^{*}$-порядок, $G^{*}$-критериальную систему класса $K$ соответственно.

Определения з.к. булевых функций Поста, з.к. четных (в том числе однородных) функций имеются в работах [1], [11] соответственно. Определения з.к. $P_{k}^{\alpha}$, $S L P_{k}, L Q_{k}, L Q_{k}^{+}, S L Q_{4}^{+}, C E_{k}, V_{4}, Q L_{4}, V Q L_{4}, V_{4}^{4} L_{4}, L L_{4}, L L_{4}^{1}, N L_{4}$ имеются в работе [6]. Там $V_{4}, V Q L_{4}, V_{4}^{4} L_{4}$ имеют обозначения $K_{4}, Q L_{4}^{V}, K L_{4}$ соответственно.

Определения 3. к. $A_{3}^{0}, J_{3}, L^{3}$ имеются в работе [10] (там $A_{3}^{0}, J_{3}$ имеют обозначения $S, S_{1}$ соответственно). Пусть $V_{4}^{\alpha}=P_{4}^{\alpha} \cap V_{4}, Q L_{4}^{\alpha}=P_{4}^{\alpha} \cap Q L_{4}, V Q L_{4}^{\alpha}=$ $P_{4}^{\alpha} \cap V Q L_{4}$,

$$
\begin{array}{r}
V_{4}^{4} L_{4}^{0}=\left\{f \in P_{k} \mid f\left(x_{1}, \ldots, x_{n}\right)=x_{i_{1}}+\ldots+x_{i_{q}}+c,\left\{i_{1}, \ldots, i_{q}\right\} \subseteq\{1, \ldots, n\},\right. \\
\left.\left|\left\{i_{1}, \ldots, i_{q}\right\}\right|=q, q \text { нечетно, } c \in E_{4}\right\} .
\end{array}
$$

Пусть

$$
\begin{aligned}
& P_{k}^{2} \vec{C}_{4}=\left\{f \in P_{k} \mid f\right. \text { сохраняет любое 2-элементное }\text { подмножество множества } \left.E_{k}\right\}, \\
& P_{k}^{2} \vec{O}_{1}=\left\{f \in P_{k}^{2} \vec{C}_{4} \mid B_{I_{i}^{2}} f \in B_{I_{i}^{2}} O_{1}, i=1, \ldots, s_{1}\right\}, \\
& P_{4}^{2} \tilde{O}_{1}=\left\{f \in P_{4}^{2} \vec{O}_{1} \mid B_{2} f \in V_{4}\right\}, \\
& P_{k}^{i} O_{1}=\left\{f \in P_{k} \mid B_{i} f \in B_{i} O_{1}, i=2, \ldots, k-1\right\}, \\
& V_{4}^{2} \tilde{D}_{1}=\left\{f \in V_{4} \mid B_{I_{i}^{2}} f \in B_{I_{i}^{2}} S_{4}, i=1, \ldots, s_{1}\right\} .
\end{aligned}
$$




\section{3. Основные результаты}

Предложение А. Для любого множества $M \subseteq P_{k}$ и любой подгруппь $G^{*} \subset$ $S\left(E_{k}\right), k \geqslant 2$, имеет место равенство $\left[G^{*}(M)\right]=[M]_{G^{*}}$.

Дбказательство. Воспользуемся формульным способом задания оператора замыкания [.] (см. [1]). Каждая формула $U$ над $G^{*}(M)$ реализует некоторую функцию $f_{U}\left(x_{1}, \ldots, x_{n}\right)$. Покажем, что для любой перестановки $\pi$ из $G^{*}$ и для любой формулы $U$ над $G^{*}(M)$ всегда имеет место включение $f_{U}^{\pi} \in\left[G^{*}(M)\right]$. Естественным образом вводится понятие глубины $l(U)$ формулы $U$ над множеством $G^{*}(M)$. Если $U$ имеет глубину 1 , т. е. $U=f_{U}\left(x_{1}, \ldots, x_{n}\right) \in G^{*}(M)$, то очевидно, что $f_{U}^{\pi} \in G^{*}(M)$. Предположим, что наше утверждение верно для всех формул $U$ глубины $l, 1 \leqslant l \leqslant r$. Рассмотрим произвольную формулу $U$ глубины $r+1$ над $G^{*}(M)$. Формула $U$ тогда имеет вид $f\left(H_{1}, \ldots, H_{m}\right)$, где $f \in G^{*}(M)$, а $H_{1}, \ldots, H_{m}$ - формулы над $G^{*}(M)$ и $\max \left(l\left(H_{1}\right), \ldots, l\left(H_{m}\right)\right) \leqslant r$. Очевидно, что $f_{U}=f\left(f_{H_{1}}, \ldots, f_{H_{m}}\right)$ и $f_{U}=f\left(\left(f_{H_{1}}\right), \ldots,\left(f_{H_{m}}\right)\right)$. Но $f^{\pi} \in G^{*}(M)$, а $f_{H_{1}}^{\pi}, \ldots, f_{H_{m}}^{\pi} \in\left[G^{*}(M)\right]$ по предположению индукции. Поэтому $f_{U}^{\pi} \in G^{*}(M)$, т.е. утверждение верно для любой $U$ с любой глубиной.

По определению $G^{*}$-замыкания очевидно, что $\left[G^{*}(M)\right] \subset[M]_{G^{*}}$. Пусть $M_{0}=$ $M$, a

$$
M_{i+1}=\left\{f \mid f \in\left[M_{i}\right] \text { или найдется } g \text { из } M_{i} \text { такая, что } g G^{*} f\right\} .
$$

Легко видеть, что

$$
[M]_{G^{*}}=\bigcup_{i=1}^{\infty} M_{i}
$$

Очевидно, что $M_{0} \subset\left[G^{*}(M)\right]$. Предположим, что $M_{i} \subset\left[G^{*}(M)\right]$. Рассмотрим произвольную функцию $h$ из $M_{i+1}$. Если $h \in\left[M_{i}\right]$, то $h \in\left[G^{*}(M)\right]$. Остается случай, когда существует $g \in M_{i}$ такая, что $g G^{*} h$, т. е. $h=g^{\sigma}$ для некоторого $\sigma \in S\left(E_{k}\right)$. По предположению индукции $g \in\left[G^{*}(M)\right]$. Из доказанного выше утверждения следует, что $h \in\left[G^{*}(M)\right]$. Тем самым доказано, что $M_{i} \in\left[G^{*}(M)\right]$, $i=1,2, \ldots$, т. е.

$$
[M]_{G^{*}}=\bigcup_{i=0}^{\infty} M_{i} \subset\left[G^{*}(M)\right]
$$

Предложение доказано.

Следствие А. Для любой подгруппы $G^{*} \subset S\left(E_{k}\right)$ и для любого $G^{*}$-з.к. $K$ из $P_{k}$, $k \geqslant 2$, имеет место равенство $\mathrm{ORD}_{G^{*}}(K)=\mathrm{ORD}(K)$.

Лемма 1. Для любого $k \geqslant 5$ пусть $f(x)$ - произвольная нетривиальная подстановка из $P S_{k}, G-m$-транзитивная подгруппа группы $P S_{k}$, где $m \geqslant[k / 2]+1$. Тогда подгруппа $[\{f(x)\}]_{G^{*}}$ 4-транзитивна при $k=2^{n}, n>2 ; 3$-транзитивна при $k=p^{n}$, где $p-$ простое число, $p \geqslant 3, n \geqslant 1 ; 2$-транзитивна при $k \neq p^{n}$, где $p-$ простое число, $n \geqslant 1$. 
Доказательство. При $5 \leqslant k \leqslant 6$, поскольку $G=A_{k}$, аналогично теореме Галуа $\left[4\right.$, стр. 115] имеем $[\{f\}]_{G^{*}} \in\left\{P A_{k}, P S_{k}\right\}$.

Рассмотрим всевозможные случаи, когда $k \geqslant 7$.

Пусть в разложении на независимые циклы (РНЦ) подстановки $f(x)$ имеется цикл $\left(a_{1} a_{2} a_{3} \ldots a_{t}\right)$ длины, не меньшей 4 . В подгруппе $G$ найдется $\varphi(x)$ такая, что $\varphi\left(a_{1}\right)=a_{2}, \varphi\left(a_{2}\right)=a_{1}, \varphi\left(a_{3}\right)=a_{3}, \varphi\left(a_{t}\right)=a_{t}$. Нетрудно видеть, что РНЦ подстановки $f_{1}(x)=f^{-1}\left(f^{\varphi}(x)\right)$ содержит цикл $\left(a_{1} a_{2} a_{3}\right)$. Отсюда легко видеть, что в $[\{f(x)\}]_{G^{*}}$ всегда найдется нетривиальная $g(x)$, РНШ которой, кроме цикла длины 1 , содержит либо только циклы длины 3 , либо только циклы длины 2.

При $k \geqslant 11$ имеем $m \geqslant 6$ и с помощью упомянутой выше теоремы Галуа легко убедиться в том, что ограничение подгруппы $[\{g(x)\}]_{G^{*}}$ на некоторое 6элементное подмножество множества $E_{k}$ изоморфно $P S_{6}$. Поэтому для любых трех пар $\left(a_{1}, a_{2}\right),\left(b_{1}, b_{2}\right),\left(c_{1}, c_{2}\right)$ из $E_{k}^{2}$ найдутся пары $\left(d_{1}, d_{2}\right),\left(e_{1}, e_{2}\right),\left(u_{1}, u_{2}\right)$ из $E_{k}^{2}$ и $\psi(x)$ из $G$ такие, что

$g\left(d_{1}\right)=d_{2}, \quad g\left(e_{1}\right)=e_{2}, \quad g\left(u_{1}\right)=u_{2}, \quad \psi\left(a_{i}\right)=d_{i}, \quad \psi\left(b_{i}\right)=e_{i}, \quad \psi\left(c_{i}\right)=u_{i}, \quad i=1,2$.

Тогда

$$
g^{\psi}\left(a_{1}\right)=a_{2}, \quad g^{\psi}\left(b_{1}\right)=b_{2}, \quad g^{\psi}\left(c_{1}\right)=c_{2},
$$

т. е. подгруппа $[\{g\}]_{G *}$ 3-транзитивна.

Аналогичным образом доказывается $l$-транзитивность подгруппы $[\{g\}]_{G^{*}}$, где $l=4$ при $k=2^{n}>11, l=3$ при $k=p^{n}>11$, где $p$ простое число, $n \geqslant 1$.

При $k=7$, если РНЦ подстановки $g(x)$ содержит циклы длины 3 , то она содержит и циклы длины 1 . Отсюда следует, что ограничение подгруппы $[\{g\}]_{G^{*}}$ на некоторое 4-элементное подмножество множества $E_{7}$ изоморфно $P S_{4}$. Поэтому можно предполагать, без ограничения общности, что $[\{g\}]_{G^{*}}$ содержит

$$
\begin{aligned}
& g_{1}(x)=\left(a_{1} a_{2}\right)\left(a_{3}\right)\left(a_{4}\right) \ldots(\ldots) \ldots(\ldots), \\
& g_{2}(x)=\left(a_{1} a_{2}\right)\left(a_{3} a_{4}\right)(\ldots) \ldots(\ldots) .
\end{aligned}
$$

Если $g_{1}(x)$ или $g_{2}(x)$ содержит циклы длины 3 , то легко видеть, что либо $g_{1}\left(g_{1}(x)\right)$ либо $g_{2}\left(g_{2}(x)\right)$ принадлежит $\left\{\left(a_{5} a_{6} a_{7}\right),\left(a_{5} a_{7} a_{6}\right)\right\}$. Нетрудно проверить, что $\left[\left\{\left(a_{5} a_{6} a_{7}\right)\right\}\right]_{G^{*}}=\left[\left\{\left(a_{5} a_{7} a_{6}\right)\right\}\right]_{G^{*}}=A_{7}$. Последняя подгруппа 3-транзитивна.

Если $g_{1}(x)$ и $g_{2}(x)$ содержит циклы длины, не превосходящей 2 , то нетрудно проверить, что

$$
g_{1}\left(g_{2}(x)\right) \in\left\{\left(a_{3} a_{4}\right),\left(a_{3} a_{4}\right)\left(a_{5} a_{6} a_{7}\right),\left(a_{3} a_{4}\right)\left(a_{5} a_{7} a_{6}\right)\right\} .
$$

Отсюда следует, что $\left(a_{3} a_{4}\right) \in\left[\left\{g_{1}\left(g_{2}(x)\right)\right\}\right]_{G^{*}}$. Но легко видеть, что $\left[\left\{\left(a_{3} a_{4}\right)\right\}\right]_{G^{*}}=$ $P S_{7}$, т. е. $[\{g(x)\}]_{G^{*}}$ 2-транзитивна.

При $k=9$ пусть $g(x)=\left(a_{1} a_{2} a_{3}\right)\left(a_{4} a_{5} a_{6}\right)\left(a_{7} a_{8} a_{9}\right)$. Поскольку $G$ 5-транзитивна, найдется $\theta(x)=\left(a_{7}\right)\left(a_{8}\right)\left(a_{9}\right)\left(a_{4} a_{5}\right)(\ldots) \ldots(\ldots)$ из $G$ такая, что

$$
g^{1}(x)=\langle\theta(x)\rangle(g(x))=\left(i_{1} i_{2} i_{3}\right)\left(a_{4} a_{5} a_{6}\right)\left(a_{7} a_{8} a_{9}\right)
$$

Тогда

$$
g^{1}((x))=\left(a_{4}\right)\left(a_{7} a_{8} a_{9}\right)(\ldots) \ldots(\ldots)
$$

Отсюда следует, что ограничение подгруппы $\left[\left\{g^{1}(g(x))\right\}\right]_{G^{*}}$ на некоторое 5элементное подмножество множества $E_{9}$ изоморфно $P S_{5}$. Легко проверить, что 
другие виды подстановки $g(x)$ также приводят к этому утверждению. Поэтому можно предполагать, без ограничения общности, что $[\{g(x)\}]_{G^{*}}$ содержит

$$
g^{2}(x)=\left(a_{1} a_{2}\right)\left(a_{3} a_{4} a_{5}\right)(\ldots)(\ldots)
$$

и из $g^{2}(x)$ суперпозицией можно получить либо $\left(a_{1} a_{2}\right)$, либо $\left(a_{3} a_{4} a_{5}\right)$. Следовательно, $\left[\left\{g^{2}(x)\right\}\right]_{G^{*}} \in\left\{P A_{9}, P S_{9}\right\}$, т. е. $[\{g(x)\}]_{G^{*}} 3$-транзитивна.

При $k=8$ пусть $g(x)=\left(a_{1} a_{2}\right)\left(a_{3} a_{4}\right)\left(a_{5} a_{6}\right)\left(a_{7} a_{8}\right)$. В $G$ найдется такая $\sigma(x)$, что

$$
g^{3}(x)=g^{\sigma}(x)=\left(a_{1}\right)\left(a_{2}\right)\left(a_{4} a_{5}\right)(\ldots)(\ldots) .
$$

Тогда

$$
g^{4}(x)=g^{3}(g(x))=\left(a_{1}\right)\left(a_{2}\right)\left(a_{3} a_{5} \ldots\right) \ldots(\ldots) .
$$

Если РНЦ $g^{4}(x)$ содержит цикл длины, не меньшей 5 , то, как уже доказано выше, в $\left[\left\{g^{4}\right\}\right]_{G^{*}}$, найдется $g^{5}(x)$, РНЦ которой содержит только циклы длин 3 и 1. Легко проверить, что другие варианты подстановки $g(x)$ также приводят к этой ситуации. Но тогда ограничение $[\{g(x)\}]_{G^{*}}$ на некоторое 5-элементное подмножество множества $E_{8}$ изоморфно $P S_{5}$. Отсюда можно предполагать, без ограничения общности, что $[\{g(x)\}]_{G^{*}}$ содержит

$$
g^{6}(x)=\left(a_{1} a_{2}\right)\left(a_{3} a_{4} a_{5}\right)(\ldots)(\ldots) .
$$

Легко видеть, что $\left[\left\{g^{6}(x)\right\}\right]_{G^{*}}$ содержит либо $\left(a_{1} a_{2}\right)$, либо $\left(a_{3} a_{4} a_{5}\right)$. Следовательно, либо $[\{g(x)\}]_{G^{*}}=P A_{8}$, либо $[\{g(x)\}]_{G^{*}}=P S_{8},[\{g(x)\}]_{G^{*}}$ 4-транзитивна. Лемма доказана.

Предложение 1. Для любого $k \geqslant 3$ пусть $G-([k / 2]+1)$-транзитивная подгрупnа группь $P S_{k}$. Тогда для любых $a_{1}, \ldots, a_{q}$ из $E_{k}$, где

$$
2 \leqslant\left|\left\{a_{1}, \ldots, a_{q}\right\}\right|=\left|\left\{b_{1}, \ldots, b_{q}\right\}\right|=q \leqslant k,
$$

в $G$ всегда найдется такая подстановка $\pi$, что

$$
\pi\left(a_{1}\right)=b_{1}, \quad \pi\left(a_{2}\right)=b_{2}, \quad\left\{\pi\left(a_{3}\right), \ldots, \pi\left(a_{q}\right)\right\}=\left\{b_{3}, \ldots, b_{q}\right\} .
$$

Доказательство очевидно.

Лемма 2. Для любого $k \geqslant 4$ пусть $f(x)$ - произвольная функиия из $P_{k}^{1(k-1)}$, $|\operatorname{Im} P| \geqslant 2, G=A_{k}$, при $k=4,5,6$ u $G-$-т-транзитивная подгруппа группь $P S_{k}, m \geqslant[k / 2]+1$ nри $k \geqslant 7$. Тогда подполугруппа $[\{f(x)\}]_{G^{*}} 2$-транзитивна.

Доказательство. Сначала покажем, что можно получить $f_{1}(x)$ из $[\{f(x)\}]_{G^{*}}$ такую, что $\left|\operatorname{Im} f_{1}\right| \geqslant 2, f_{1}(a)=a$ для каждого $a \in \operatorname{Im} f_{1}(x)$. Если существует хотя бы одно $r$-элементное подмножество $M \subset E_{k}$ такое, что $r \geqslant 2, f$ сохраняет $M$ и ограничение $f$ на $M$ принимает ровно $r$ различных значений, то легко убедиться в том, что $k$-кратная итерация $f(f(\ldots(f(x)) \ldots))$ является искомой $f_{1}$. В противном случае $f(x)$ должна быть такой, что $f\left(i_{1}\right)=i_{2}, f\left(i_{2}\right)=i_{3}, \ldots, f\left(i_{k-1}\right)=i_{k}$, $f\left(i_{k}\right)=i_{k}$, где $\left\{i_{1}, \ldots, i_{k}\right\}=E_{k}$. Пусть $f^{1}(x)=f(f(\ldots(f(x)) \ldots))-(k-2)$ кратная итерация $f$. Тогда $f^{1}\left(i_{1}\right)=i_{k-1}, f^{1}\left(i_{2}\right)=\ldots=f^{1}\left(i_{k}\right)=i_{k}$. В $G$ найдется 
такая $\varphi(x)$, что $\varphi\left(i_{1}\right)=i_{k-1}, \varphi\left(i_{k-1}\right)=i_{1}$. Пусть $\varphi^{-1}\left(i_{k}\right)=i_{p}$. Очевидно, что $i_{p} \notin\left\{i_{1}, i_{k-1}\right\}$. Пусть $f^{2}(x)=\langle\varphi\rangle\left(f^{1}(x)\right)$. Легко проверить, что $f^{2}\left(i_{k-1}\right)=i_{1}$, $f^{2}\left(i_{1}\right)=\ldots=f^{2}\left(i_{k-2}\right)=f^{2}\left(i_{k}\right)=i_{p}$ и для $f^{3}(x)=f^{1}\left(f^{2}(x)\right)$ имеем $f^{2}\left(i_{k-1}\right)=i_{k-1}$, $f^{3}\left(i_{k}\right)=i_{k}$. Возвращаемся к знакомой ситуации. Пусть

$$
f_{1}(x)=\left(\begin{array}{cccc}
M_{1} & M_{2} & \ldots & M_{l} \\
a_{1} & a_{2} & \ldots & a_{l}
\end{array}\right)
$$

где $M_{1} \cup \ldots \cup M_{l}=E_{k},\left\{a_{1}, \ldots, a_{l}\right\}-l$-элементное подмножество множества $E_{k}$, $2 \leqslant l \leqslant k-1, f\left(a_{i}\right)=a_{i}, i=1, \ldots, l$. Пусть $\rho\left(f_{1}\right)-$ ранг [5] подстановки $f_{1}$. Пусть $\rho\left(f_{1}\right)=l \geqslant 3$. Без ограничения общности можно предполагать, что $\left|M_{2}\right| \geqslant 2$ и $M_{2}$ содержит $b_{2} \neq a_{2}$. При $k=4$ легко видеть, что $G=A_{4}$ и в $G$ найдется такая $\psi(x)$, что $\psi(x)=\left(a_{1} a_{2}\right)\left(b_{2} a_{3}\right)$. Пусть $f_{1}^{1}(x)=f_{1}^{\psi}(x)$. Нетрудно проверить, что для $f_{2}(x)=f_{1}^{1}\left(f_{1}(x)\right)$ имеем $\rho\left(f_{2}\right)=2$. При $k \geqslant 5$ в $G$ найдется такая $\psi(x)$, что $\psi\left(a_{1}\right)=a_{1}, \psi\left(a_{2}\right)=a_{2}, \psi\left(a_{3}\right)=b_{2}$. Пусть $f_{2}(x)=f_{1}^{1}\left(f_{1}(x)\right)$. Легко видеть, что $2 \leqslant \rho\left(f_{2}\right) \leqslant \rho\left(f_{1}\right)-1$. Эти соображения позволяют убедиться в том, что в $[\{f\}]_{G^{*}}$ всегда найдется такая функция $f_{3}(x)$, что $\rho\left(f_{3}\right)=2$ и $f_{3}$ неподвижна на $\operatorname{Im} f_{3}$. Предположим, что

$$
f_{3}(x)=\left(\begin{array}{cc}
M_{1} & M_{2} \\
a_{1} & a_{2}
\end{array}\right)
$$

где $a_{1} \in M_{1}, a_{2} \in M_{2}, k_{1}=\left|M_{1}\right| \geqslant k_{2}=\left|M_{2}\right| \geqslant 1, k_{1}+k_{2}=k$. Как в [6], пусть $\lambda^{f_{3}}=\left(k_{1}, k_{2}\right)$. Покажем, что $[\{f\}]_{G^{*}}$ содержит

$$
H_{f_{3}}^{0}=\left\{g(x) \in P_{k}^{1[2]} \mid \lambda^{g}=\left(k_{1}, k_{2}\right) \text { и } g \text { неподвижна на } \operatorname{Im} g=\operatorname{Im} f_{3}\right\} \text {. }
$$

При $k=4, k_{1}=3, k_{2}=1$ без ограничения общности можно предполагать, что

$$
f_{3}(x)=\left(\begin{array}{llll}
a_{1} & b_{1} & b_{2} & a_{2} \\
a_{1} & a_{1} & a_{1} & a_{2}
\end{array}\right) .
$$

Тогда $H_{f_{3}}^{0}=\left\{f_{3}, g_{3}\right\}$, где

$$
g_{3}(x)=\left(\begin{array}{llll}
a_{2} & b_{1} & b_{2} & a_{1} \\
a_{2} & a_{2} & a_{2} & a_{1}
\end{array}\right)
$$

Легко видеть, что $g=f_{3}^{\pi_{1}}$, где $\pi_{1}(x)=\left(b_{1} b_{2}\right)\left(a_{1} a_{2}\right)$.

При $k=4, k_{1}=2, k_{2}=2$ будем предполагать, что

$$
f_{3}(x)=\left(\begin{array}{llll}
a_{1} & b_{1} & a_{2} & b_{2} \\
a_{1} & a_{1} & a_{2} & a_{2}
\end{array}\right)
$$

Тогда $H_{f_{3}}^{0}=\left\{f_{3}, g_{4}\right\}$, где

$$
g_{4}(x)=\left(\begin{array}{llll}
a_{1} & b_{2} & a_{2} & b_{1} \\
a_{1} & a_{1} & a_{2} & a_{2}
\end{array}\right) .
$$

Пусть $\pi_{2}(x)=\left(a_{1} b_{2} a_{2}\right)\left(b_{1}\right), g_{4}^{1}=f_{3}^{\pi_{2}}(x), g_{4}^{2}=g_{4}^{1}\left(f_{3}(x)\right), \pi_{3}(x)=\left(b_{2} a_{1} b_{1}\right)\left(a_{2}\right), g_{4}^{3}(x)=$ $\left\langle\pi_{3}(x)\right\rangle\left(g_{4}^{2}(x)\right)$. Тогда $g_{4}(x)=f_{3}\left(g_{4}^{3}(x)\right)$.

При $k \geqslant 5$ без ограничения общности можно предполагать, что

$$
f_{3}(x)=\left(\begin{array}{ccccccccc}
a_{1} & b_{1} & b_{2} & \ldots & b_{p} & a_{2} & c_{1} & \ldots & c_{q} \\
a_{1} & a_{1} & a_{1} & \ldots & a_{1} & a_{2} & a_{2} & \ldots & a_{2}
\end{array}\right)
$$


где $k_{1}=p+1, k_{2}=q+1$. Чтобы доказать, что $\left[\left\{f_{3}\right\}\right]_{G^{*}} \supseteq H_{f_{3}}^{0}$, достаточно доказать, что $\left[\left\{f_{3}\right\}\right]_{G^{*}}$ содержит

$$
\begin{aligned}
g_{5}(x) & =\left(\begin{array}{cccccccc}
a_{1} & b_{i_{1}} & \ldots & b_{i_{p}} & a_{2} & c_{i_{1}} & \ldots & c_{i_{q}} \\
a_{1} & a_{1} & \ldots & a_{1} & a_{2} & a_{2} & \ldots & a_{2}
\end{array}\right), \\
g_{6}(x) & =\left(\begin{array}{ccccccccc}
a_{2} & b_{j_{1}} & \ldots & b_{j_{p}} & a_{1} & c_{j_{1}} & \ldots & c_{j_{q}} \\
a_{2} & a_{2} & \ldots & a_{2} & a_{1} & a_{1} & \ldots & a_{1}
\end{array}\right)
\end{aligned}
$$

для произвольных $b_{i_{1}}, \ldots, b_{i_{p}}, c_{i_{1}}, \ldots, c_{i_{q}}, b_{j_{1}}, \ldots, b_{j_{p}}, c_{j_{1}}, \ldots, c_{j_{q}}$ из $E_{k} \backslash\left\{a_{1}, a_{2}\right\}$. Так как $m \geqslant[k / 2]+1$, в группе $G$ всегда найдутся $\pi_{5}(x), \pi_{6}(x)$ такие, что.

$$
\begin{aligned}
\pi_{5}(x) & =\left(\begin{array}{llllllll}
a_{1} & a_{2} & c_{i_{1}} & \ldots & c_{i_{q}} & * & \ldots & * \\
a_{1} & a_{2} & c_{1} & \ldots & c_{q} & * & \ldots & *
\end{array}\right), \\
\pi_{6}(x) & =\left(\begin{array}{llllllll}
a_{1} & a_{2} & c_{i_{1}} & \ldots & c_{i_{q}} & * & \ldots & * \\
a_{2} & a_{1} & c_{1} & \ldots & c_{q} & * & \ldots & *
\end{array}\right) .
\end{aligned}
$$

(Знак * означает, что значение числа на месте этого знака не определено.) Нетрудно проверить, что $\left(f_{3}^{\pi_{5}}(x)\right)=g_{5}(x),\left(f_{3}^{\pi_{6}}(x)\right)=g_{6}(x)$. Для произвольных различных $a_{i_{1}}, a_{i_{2}}$ из $E_{k}$ пусть

$$
\pi_{7}(x)=\left(\begin{array}{ccc}
a_{1} & a_{i_{2}} & \cdots \\
a_{1} & a_{2} & \cdots
\end{array}\right) \in G
$$

для $g_{7}=\left(f_{3}^{\pi_{7}}(x)\right)$ имеем $\operatorname{Im} g_{7}(x)=\left\{a_{i_{1}}, a_{i_{2}}\right\}, g_{7}\left(a_{i_{1}}\right)=a_{i_{1}}, g_{7}\left(a_{i_{2}}\right)=a_{i_{2}}$. Тем самым доказано, что для любых $k_{1}, k_{2}$, где $k_{1} \geqslant 1, k_{2} \geqslant 1, k_{1}+k_{2}=1,\left[\left\{f_{3}\right\}\right]_{G^{*}}$ содержит

$$
H^{0}\left(k_{1}, k_{2}\right)=\left\{g \in P_{k}^{1[2]} \mid \lambda^{g}=\left(k_{1}, k_{2}\right), g \text { неподвижна на } \operatorname{Im} g\right\} .
$$

Теперь докажем, что

$$
\left[\left\{f_{3}\right\}\right]_{G^{*}} \supseteq H\left(k_{1}, k_{2}\right)=\left\{h(x) \in P_{k}^{1[2]} \mid \lambda^{h}=\left(k_{1}, k_{2}\right)\right\} .
$$

Рассмотрим сначала случай $k_{1}=k-1$. Без ограничения общности можно предполагать, что

$$
f_{3}(x)=\left(\begin{array}{ccccc}
0 & 1 & 2 & \ldots & k-1 \\
0 & 1 & 1 & \ldots & 1
\end{array}\right) .
$$

Нам достаточно показать, что для любого $i, 1<i \leqslant k-1,\left[\left\{f_{3}\right\}\right]_{G^{*}}$ содержит

$$
\begin{aligned}
f_{3}^{1}(x) & =\left(\begin{array}{ccccc}
0 & 1 & 2 & \ldots & k-1 \\
1 & 0 & 0 & \ldots & 0
\end{array}\right), \\
f_{3}^{2}(x) & =\left(\begin{array}{ccccc}
0 & 1 & 2 & \ldots & k-1 \\
1 & 0 & 1 & \ldots & 1
\end{array}\right), \\
f_{3}^{3}(x) & =\left(\begin{array}{cccccccc}
0 & 1 & \ldots & i-1 & i & i+1 & \ldots & k-1 \\
1 & 1 & \ldots & 1 & 0 & 1 & \ldots & 1
\end{array}\right), \\
f_{3}^{4}(x) & =\left(\begin{array}{cccccccc}
0 & 1 & \ldots & i-1 & i & i+1 & \ldots & k-1 \\
0 & 0 & \ldots & 0 & 1 & 0 & \ldots & 0
\end{array}\right) .
\end{aligned}
$$

В $G$ найдутся $\sigma_{i}(x), i=1, \ldots, 4$, такие, что если $k \geqslant 5$, то $\sigma_{1}(0)=\sigma_{4}(0)=1$, $\sigma_{1}(1)=\sigma_{4}(1)=0, \sigma_{1}(i)=\sigma_{4}(i)=i, \sigma_{2}(0)=i, \sigma_{2}(i)=0, \sigma_{2}(1)=1, \sigma_{3}(1)=i$, 
$\sigma_{3}(i)=1$, а если $k=4$ (тогда $G=A_{4}$ ), то $\sigma_{1}(x)=(01)(23), \sigma_{2}(x)=(1)(i 0 t)$, $\sigma_{3}(x)=(1 i)(0 t), \sigma_{4}(x)=(i)(01 t)$, где $t \in\{2,3\} \backslash\{i\}$. Легко проверить, что

$$
\begin{aligned}
& h_{1}(x)=f_{3}^{\sigma_{1}}(x)=\left(\begin{array}{llllc}
0 & 1 & 2 & \ldots & k-1 \\
1 & 0 & 0 & \ldots & 0
\end{array}\right), \\
& h_{2}(x)=h_{1}^{\sigma_{2}}(x)=\left(\begin{array}{ccccc}
0 & 1 & 2 & \ldots & k-1 \\
i & 0 & i & \ldots & i
\end{array}\right) \text {, } \\
& h_{3}(x)=h_{2}^{\sigma_{3}}(x)=\left(\begin{array}{cccccccc}
0 & 1 & \ldots & i-1 & i & i+1 & \ldots & k-1 \\
1 & 1 & \ldots & i & 0 & 1 & \ldots & 1
\end{array}\right) \\
& h_{4}(x)=h_{3}^{\sigma_{4}}(x)=\left(\begin{array}{cccccccc}
0 & 1 & \ldots & i-1 & i & i+1 & \ldots & k-1 \\
0 & 0 & \ldots & 0 & i & 0 & \ldots & 0
\end{array}\right) \text {, } \\
& f_{3}^{4}(x)=f_{3}\left(h_{4}(x)\right), \quad f_{3}^{2}(x)=f_{3}^{4}\left(h_{2}(x)\right), \quad f_{3}^{3}(x)=f_{3}^{2}\left(f_{3}^{4}(x)\right), \quad f_{3}^{1}(x)=f_{3}^{2}\left(f_{3}(x)\right) .
\end{aligned}
$$

Рассмотрим случаи, когда $k_{1} \geqslant k_{2} \geqslant 2$. Без ограничения общности можно предполагать, что

$$
f_{3}(x)=\left(\begin{array}{cccccccc}
0 & a_{1} & \ldots & a_{k_{1}-1} & 1 & b_{1} & \ldots & b_{k_{2}-1} \\
0 & 0 & \ldots & 0 & 1 & 1 & \ldots & 1
\end{array}\right) .
$$

В силу включения $\left[\left\{f_{3}\right\}\right]_{G^{*}} \supseteq H^{0}\left(k_{1}, k_{2}\right)$ нам достаточно доказать, что для любого $a_{s} \in\left\{a_{1}, \ldots, a_{k_{1}-1}\right\}$ множество $\left[\left\{f_{3}\right\}\right]_{G^{*}}$ содержит функции

$$
\begin{aligned}
f_{3,1}(x) & =\left(\begin{array}{cccccccccccc}
0 & a_{1} & \ldots & a_{k_{1}-1} & 1 & b_{1} & \ldots & b_{k_{2}-1} \\
1 & 1 & \ldots & 1 & 0 & 0 & \ldots & 0
\end{array}\right), \\
f_{3,2}(x) & =\left(\begin{array}{ccccccccccccc}
0 & 1 & a_{1} & \ldots & a_{s-1} & a_{s+1} & \ldots & a_{k_{1}-1} & a_{s} & b_{1} & \ldots & b_{k_{2}-1} \\
0 & 0 & 0 & \ldots & 0 & 0 & \ldots & 0 & 1 & 1 & \ldots & 1
\end{array}\right), \\
f_{3,3}(x) & =\left(\begin{array}{cccccccccccc}
0 & 1 & a_{1} & \ldots & a_{s-1} & a_{s+1} & \ldots & a_{k_{1}-1} & a_{s} & b_{1} & \ldots & b_{k_{2}-1} \\
1 & 1 & 1 & \ldots & 1 & 1 & \ldots & 1 & 0 & 0 & \ldots & 0
\end{array}\right) .
\end{aligned}
$$

В $G$ найдется

$$
\sigma^{1}(x)=\left(\begin{array}{cccccccc}
\ldots & a_{1} & b_{1} & 1 & b_{2} & \ldots & b_{k_{2}-2} & b_{k_{2}-1} \\
\ldots & 0 & 1 & b_{2} & b_{3} & \ldots & b_{k_{2}-1} & b_{1}
\end{array}\right)
$$

такая, что

$$
h^{1}(x)=f_{3}^{\sigma^{1}}(x)=\left(\begin{array}{cccccccccc}
0 & a_{1} & a_{2} & \ldots & a_{k_{1}-1} & 1 & b_{1} & b_{2} & \ldots & b_{k_{2}-1} \\
a_{1} & a_{1} & a_{1} & \ldots & a_{1} & b_{1} & b_{1} & b_{1} & \ldots & b_{1}
\end{array}\right) .
$$

Справедливо включение

$$
\left[\left\{f_{3}\right\}\right]_{G^{*}} \supseteq H^{0}\left(k_{1}, k_{2}\right) \ni h_{2}(x)=\left(\begin{array}{ccccccc}
0 & a_{1} & 0 & b_{1} & * & \ldots & * \\
1 & 1 & 0 & 0 & * & \ldots & *
\end{array}\right) .
$$

Тогда

$$
f_{3,1}(x)=h^{2}\left(h^{1}(x)\right) .
$$

При $k=4$ для $\sigma^{2}(x)=(1)\left(a_{1} 0 b_{1}\right) \in G$

$$
h^{3,1}(x)=f_{3}^{\sigma_{2}}(x)=\left(\begin{array}{cccc}
0 & 1 & a_{1} & b_{1} \\
a_{1} & a_{1} & 1 & 1
\end{array}\right) .
$$


Очевидно, что

$$
h^{3,2}(x)=\left(\begin{array}{llll}
a_{1} & b_{1} & 1 & 0 \\
a_{1} & a_{1} & 0 & 0
\end{array}\right) \in H^{0}\left(k_{1}, k_{2}\right) .
$$

Пусть

$$
h^{3}(x)=h^{3,2}\left(h^{3,1}(x)\right)=\left(\begin{array}{cccc}
0 & 1 & a_{1} & b_{1} \\
a_{1} & a_{1} & 0 & 0
\end{array}\right) .
$$

При $k \geqslant 5 G$ содержит

$$
\sigma^{3}(x)=\left(\begin{array}{cccccccc}
* & \ldots & * & 0 & a_{s} & b_{1} & \ldots & b_{k_{2}-1} \\
* & \ldots & * & 0 & 1 & b_{1} & \ldots & b_{k_{2}-1}
\end{array}\right) .
$$

Тогда пусть

$$
\begin{aligned}
h^{3}(x)=f_{3,1}^{\sigma^{3}}(x) & \\
& =\left(\begin{array}{cccccccccccc}
0 & 1 & a_{1} & \ldots & a_{s-1} & a_{s+1} & \ldots & a_{k_{1}-1} & a_{s} & b_{1} & \ldots & b_{k_{2}-1} \\
a_{s} & a_{s} & a_{s} & \ldots & \ldots & \ldots & \ldots & a_{s} & 0 & 0 & \ldots & 0
\end{array}\right) .
\end{aligned}
$$

При $k=4$ для $\sigma^{4}(x)=(0)\left(a_{1} 1 b_{1}\right)$

$$
h^{4,1}(x)=f_{3,1}^{\sigma^{4}}(x)=\left(\begin{array}{cccc}
0 & b_{1} & a_{1} & 1 \\
a_{1} & a_{1} & 0 & 0
\end{array}\right) .
$$

Очевидно, что

$$
h^{4,2}(x)=\left(\begin{array}{cccc}
1 & a_{1} & 0 & b_{1} \\
1 & 1 & 0 & 0
\end{array}\right) \in H^{0}\left(k_{1}, k_{2}\right)
$$

Пусть

$$
h^{4}(x)=h^{4,2}\left(h^{4,1}(x)\right)=\left(\begin{array}{cccc}
0 & a_{1} & * & * \\
1 & 0 & * & *
\end{array}\right) .
$$

При $k \geqslant 5$ подгруппа $G$ содержит

$$
\sigma^{5}(x)=\left(\begin{array}{ccccccccc}
* & \ldots & * & a_{s} & 0 & 1 & b_{2} & \ldots & b_{k_{2}-1} \\
* & \ldots & * & b_{1} & 0 & 1 & b_{2} & \ldots & b_{k_{2}-1}
\end{array}\right) .
$$

Тогда пусть

$$
h^{4}(x)=f_{3,1}^{\sigma^{5}}(x)\left(\begin{array}{ccccc}
0 & a_{s} & * & \ldots & * \\
1 & 0 & * & \ldots & *
\end{array}\right) .
$$

Таким образом, при $k \geqslant 4$ легко проверить, что

$$
h^{4}\left(h^{3}(x)\right)=f_{3,2}(x), \quad f_{3,1}(x)\left(f_{3,2}(x)\right)=f_{3,3}(x) .
$$

Итак, доказано, что $H\left(k_{1}, k_{2}\right) \subseteq\left[\left\{f_{3}\right\}\right]_{G^{*}}$. Отсюда следует, что $\left[\left\{f_{3}\right\}\right]_{G^{*}}$ 2-транзитивна. Лемма доказана.

Из доказательства леммы 2 нетрудно извлечь следующую лемму.

Лемма 3. Для любого $k \geqslant 4$ пусть $f(x)-$ произвольная функция из $P_{k}^{1(k-1)}, G-$ $m$-транзитивная подгруппа из $P S_{k}, m \geqslant[k / 2]+1$. Тогда $Q E_{k} \subseteq[\{f\}]_{G^{*}}$. 
Следующая лемма является очевидной модификацией леммы 5 из [6].

Лемма 4. Для любьх $k, l, k \geqslant 5, k \geqslant l \geqslant 3$, пусть $f\left(x_{1}, \ldots, x_{n}\right)-$ произвольная существенная функция из $P_{k}$, принимающая $l$ значеий, $G-$ произвольная $m$-транзитивная подгруппа группь $P S_{k}, m \geqslant[k / 2]+1$. Тогда $\left[\{f\} \cup C F_{k}\right]_{G^{*}}$ содержит 2-транзитивную подполугруппу полугруппы $P_{k}^{1(2)}$ или s-транзитивную nодәрyпny $\widehat{G}$ гpynnbl $P S_{k}$, где $s \geqslant 4 n p u k=2^{u}, u>2 ; s \geqslant 3 n p u k=p^{u}, p-$ простое число, $p \geqslant 3, u \geqslant 1 ; s \geqslant 2$ nрu $k \neq p^{u}, p-$ простое число, $u \geqslant 1$.

Теорема 1. Пусть $k \geqslant 5, G-$ произвольная $m$-транзитивная подгруппа группь $P S_{k}, m \geqslant[k / 2]+1$. Система функций $\Sigma$ из $P_{k} G^{*}$-полна тогда и только тогда, когда она целиком не содержится ни в одном из двух $G^{*}-$ з. к. $P_{k}^{\alpha} u S L P_{k}$.

Доказательство. Необходимость очевидна. Докажем достаточность: Пусть $\Sigma$ целиком не содержится ни в одном из классов $P_{k}^{\alpha}$ и $S L P_{k}$. Тогда $\Sigma$ содержит функции $f\left(x_{1}, \ldots, x_{n_{1}}\right), f\left(x_{1}, \ldots, x_{n_{2}}\right)$, не принадлежащие $P_{k}^{\alpha}, S L P_{k}$. Так как $h_{1}(x)=f_{1}(x, \ldots, x) \neq x$, то возможно только два случая. $1 . \operatorname{Im} h_{1}(x)=E_{k}$. Тогда $h_{1}(x)$ - нетривиальная подстановка группы $P S_{k}$. По лемме 4 подгруппа $\left[\left\{h_{1}(x)\right\}\right]_{G^{*}}$

( $\alpha)$ 4-транзитивна при $k=2^{u}, u>2$;

( $\beta$ ) 3-транзитивна при $k=p^{u}, p-$ простое число, $p \geqslant 3, u \geqslant 1$;

( $\gamma$ ) 2-транзитивна при $k \neq p^{u}, p$ - простое число, $u \geqslant 1$.

По теореме 3.1.2 работы [7] подгруппа $\left[\left\{h_{1}(x)\right\}\right]_{G^{*}}$ базисна. Поэтому

$$
\left[\left\{h_{1}(x)\right\}, f_{2}\left(x_{1}, \ldots, x_{n_{2}}\right)\right]_{G^{*}}=P_{k} .
$$

2. $\left|\operatorname{Im} h_{1}(x)\right|<k$. Тогда в силу леммы 3 справедливо включение $C E_{k} \subseteq$ $\left[\left\{h_{1}(x)\right\}\right]_{G^{*}}$. По лемме $4\left[\left\{f_{2}\right\} \cup C E_{k}\right]_{G^{*}}$ содержит либо подгруппу $\widehat{G}$, удовлетворяющую условиям $\alpha, \beta, \gamma$, либо 2 -транзитивную подполугруппу $F$ полугруппы $P_{k}^{1(2)}$. Ясно, что в первом варианте имеем $\left[\left\{f_{2}\right\} \cup \widehat{G}\right]_{G^{*}}=P_{k}$ согласно теореме 3.1 .2 работы [7]. Во втором варианте согласно работе [8] $P_{2}^{(k)} \subseteq\left[\left\{f_{2}\right\} \cup F\right]_{G^{*}}$. Поскольку $m \geqslant[k / 2]+1$, то для любых двух $l$-элементных подмножеств $A=\left\{a_{1}, \ldots, a_{l}\right\}$, $B=\left\{b_{1}, \ldots, b_{l}\right\}$ множества $E_{k}$ существует подстановка $\varphi$ из $G$ такая, что $B=\left\{\varphi^{-1}\left(a_{1}\right), \ldots, \varphi^{-1}\left(a_{l}\right)\right\}$, т. е., если функция $f_{2}$ принимает на $\Theta=C_{1} \times \ldots \times C_{n_{2}}$, $C_{i} \subset E_{k},\left|C_{i}\right|=l-1, i=1, \ldots, n_{2}, l$ заданных значений $a_{1}, \ldots, a_{l}$, то функция $\langle\varphi\rangle(f)$ тоже существенна и на $\Theta^{\prime}=C_{1}^{\prime} \times \ldots \times C_{n_{2}}^{\prime}, C_{i}^{\prime}=\varphi^{-1}\left(C_{i}\right), i=1, \ldots, n_{2}$, принимает $l$-значений $b_{1}, \ldots, b_{l}$. Согласно работе [3] это приводит к соотношению $P_{k}^{(l)} \subset\left[P_{k}^{(l-1)} \cup f_{2}\right]_{G^{*}}$. Продолжая такое рассуждение, получим равенство $P_{k}=\left[P_{k}^{(2)} \cup\left\{f_{2}\right\}\right]_{G^{*}}=\left[F \cup\left\{f_{2}\right\}\right]_{G^{*}}$. Теорема доказана.

Предложение 2. Пусть $k \geqslant 3, i \geqslant 2, f\left(x_{1}, \ldots, x_{n}\right)-$ произвольная функция из $P_{k}^{i} O_{1}$. Тогдa ORD $\left(\left[\left\{f, l_{i+1}\right\}\right]\right) \leqslant k$. 
Доказательство. Следуя работе [9], для любого $n \geqslant 1$ через $\Delta_{n}$ обозначим множество всех отношений эквивалентности на множестве $\{1,2, \ldots, n\}$. При $1 \leqslant m \leqslant n$ через $\Delta_{n}^{m}$ обозначим подмножество $\Delta_{n}$, состоящее из всех эквивалентностей, которые имеют ровно $m$ классов эквивалентности. Пусть $\delta \in \Delta_{n}^{m}$, а функция $\varphi:\{1, \ldots, n\} \rightarrow\{1, \ldots, n\}$ по каждому числу $j, 1 \leqslant j \leqslant n$, дает наименьшее число из класса эквивалентности, содержащего $j$. Для любой функции $f\left(x_{1}, \ldots, x_{n}\right)$ и любой эквивалентности $\delta \in \Delta_{n}^{m}$ через $f^{\delta}$ обозначим функцию $f\left(x_{\varphi(1)}, \ldots, x_{\varphi(n)}\right)$, существенно зависящую, очевидно, не более чем от $m$ переменных. Для любого $a=\left(a_{1}, \ldots, a_{n}\right) \in E_{k}^{n}$ положим

$$
\operatorname{Ker}(a)=\left\{(i, j): a_{i}=a_{j}\right\} .
$$

Очевидно, что $\operatorname{Ker}(a) \in \Delta_{n}$. Пусть

$$
\bigcup_{m=i+1}^{k} \Delta_{n}^{m}=\left\{\delta_{1}, \ldots, \delta_{t}\right\}
$$

Рассуждая аналогично доказательству леммы 1 работы [9], заключаем, что всегда найдется $g\left(x_{1}, \ldots, x_{n}, y_{1}, \ldots, y_{t}\right)$ из $S_{k}^{i} O_{1}^{*}=\left[\left\{l_{i+1}\right\}\right]$ такая, что

$$
\vec{B}_{i} g\left(x_{1}, \ldots, x_{j}, \ldots, x_{n}, y_{1}, \ldots, y_{t}\right)=\vec{B}_{i} g\left(x_{1}, \ldots, x_{n}\right)=x_{u} \in\left\{x_{1}, \ldots, x_{n}\right\}
$$

и если набор $a=\left(a_{1}, \ldots, a_{n}\right) \in E_{k}^{n}$ содержит более $i$ различных элементов, а $\operatorname{Ker}(a)=\delta_{p}$, то

$$
g\left(a_{1}, \ldots, a_{n}, y_{1}, \ldots, y_{p}, \ldots, y_{t}\right)=y_{p} .
$$

Легко проверить, что

$$
f\left(x_{1}, \ldots, x_{n}\right)=g\left(x_{1}, \ldots, x_{n}, f^{\delta_{1}}\left(x_{1}, \ldots, x_{n}\right), \ldots, f^{\delta_{t}}\left(x_{1}, \ldots, x_{n}\right)\right) .
$$

Отсюда следует, что $\operatorname{ORD}\left(\left[\left\{f, l_{i+1}\right\}\right]\right) \leqslant k$. Предложение доказано.

Теорема 2. Для любого $k \geqslant 3$ и для любой $m$-транзитивной подгруппы $G$ группьг $P S_{k}$, где $m \geqslant[k / 2]+1$ семейстөо $L_{G^{*}}\left(P_{k}\right)$ всех $G^{*}$-классов $k$-значной логики конечно и каждый $G^{*}$-класс имеет конечный базис.

Доказательство. Нам достаточно доказать, что для любой функции $f\left(x_{1}, \ldots, x_{n}\right)$, существенно зависящей от всех $n$ переменных, $n \geqslant k^{3}, G^{*}$-порядок класса $[\{f\}]_{G^{*}}$ не больше $k^{3}$.

Если $|\operatorname{Im} f(x, \ldots, x)| \leqslant k-1$, то $C E_{k} \subset[\{f\}]_{G^{*}}$ согласно лемме 3. Если $\left|\operatorname{Im} f\left(x_{1}, \ldots, x_{n}\right)\right|=l \geqslant 3$, то так же, как в доказательстве теоремы 1 , находим, что $[\{f\}]_{G^{*}} \equiv P_{k}^{(l)}$. Отсюда легко следует, что ORD $[\{f\}]_{G^{*}}=2$.

Предположим, что $\left|\operatorname{Im} f\left(x_{1}, \ldots, x_{n}\right)\right|=2$. Если $f$ не квазилинейна, то так же, как в доказательстве предложения 9 работы $[6]$, находим, что $[\{f\}]_{G^{*}}=P_{k}^{(2)}$. Отсюда легко следует, что $\mathrm{ORD}\left([\{f\}]_{G^{*}}\right)=2$. Если $f$ квазилинейна и $f \notin L Q_{k}^{+}$ при четном $k$ то так же, как в доказательствах предложений 7,8 работы.[6], находим, что $[\{f\}]_{G^{*}} \equiv L Q_{k}$. Отсюда легко следует, что $\mathrm{ORD}\left([\{f\}]_{G^{*}}\right)=2$. Если $f \in L Q_{k}^{+}$, то аналогично предложению 8 работы $[6]$ находим, что $[\{f\}]_{G^{*}} \equiv L Q_{k}^{+}$. Отсюда легко следует, что $\mathrm{ORD}\left([\{f\}]_{G^{*}}\right)=2$. 
Предположим, что $f(x, \ldots, x)=x$ и рассмотрим всевозможные случаи относительно этого предположения.

Случай I. $\vec{B}_{u} f\left(x_{1}, \ldots, x_{n}\right)=x_{v}, k-1 \geqslant u \geqslant 2, v \in\{1, \ldots, n\}$, $I_{k, u+1} f\left(x_{1}, \ldots, x_{n}\right) \neq x_{v}$. Подходящей подстановкой переменных из $f\left(x_{1}, \ldots, x_{n}\right)$ можно получить такую $g\left(x_{1}, \ldots, x_{u+1}\right)$, что

$$
\vec{B}_{u} g\left(x_{1}, \ldots, x_{u+1}\right)=x_{1}, \quad g\left(a_{1}, \ldots, a_{u+1}\right)=a_{t} \neq a_{1}
$$

для некоторого набора $\tilde{a}=\left(a_{1}, \ldots, a_{u+1}\right) \in I_{k, u+1}^{u+1}$.

1. Пусть $g(\tilde{b}) \in M_{\tilde{b}}$ для любого набора $\tilde{b}=\left(b_{1}, \ldots, b_{u+1}\right) \in I_{k, u+1}^{u+1}$. Действие группы $G$ разбивает $I_{k, u+1}^{u+1}$ на непересекающиеся орбиты $O_{1}^{*}, \ldots, O_{\mu}^{*}$. Можно предполагать, без ограничения общности, что $a_{t} \in\left\{a_{2}, \ldots, a_{u+1}\right\}$, $\left(a_{1}, \ldots, a_{u+1}\right) \in O_{1}^{*}$. Пусть

$$
g_{1}\left(x_{1}, \ldots, x_{u+1}\right)=g\left(x_{1}, \ldots, x_{t-1}, g\left(x_{1}, \ldots, x_{t}\right), x_{t+1}, \ldots, x_{u+1}\right) .
$$

Очевидно, что

$$
g_{1}\left(a_{1}, \ldots, a_{u+1}\right)=a_{t}, \quad g_{1}\left(b_{1}, \ldots, b_{u+1}\right) \in\left\{b_{1}, b_{t}\right\}
$$

для любого набора $\tilde{b}_{1}=\left(b_{1}, \ldots, b_{u+1}\right) \in I_{k, u+1}^{u+1}$. Пусть для некоторого $\tilde{c}=$ $\left(c_{1}, \ldots, c_{u+1}\right) \in O_{1}^{*} \backslash\{\tilde{a}\}$ справедливо равенство $g_{1}(\tilde{c})=c_{1}$, тогда в $G^{*}\left(g_{1}\right)$ найдется такая $g_{2}\left(x_{1}, \ldots, x_{u+1}\right)$, что $\vec{B}_{u} g_{2}=x_{1}, g_{2}\left(c_{1}, \ldots, c_{u+1}\right)=c_{t}$. Пусть

$$
g_{3}\left(x_{1}, \ldots, x_{u+1}\right)=g_{2}\left(g_{1}\left(x_{1}, \ldots, x_{u+1}\right), x_{2}, \ldots, x_{u+1}\right) .
$$

Легко видеть, что $\vec{B}_{u} g_{3}=x_{1}, g_{3}\left(b_{1}, \ldots, b_{u+1}\right)=b_{t}$ для любого $\tilde{b} \in I_{k, u+1}^{u+1}$ такого, что $g_{1}(\tilde{b})=b_{t}$. Кроме того $g_{3}(\tilde{c})=c_{t}$. Продолжая такую процедуру из $g_{1} G^{*}$-замыканием можно получить $g_{\tau_{1}}\left(x_{1}, \ldots, x_{u+1}\right)$ такую, что $\vec{B}_{u} g_{\tau_{1}}=x_{1}$, $g_{\tau_{1}}\left(x_{1}, \ldots, x_{u+1}\right) \equiv x_{t}$ на $O_{1}^{*}, g_{\tau_{1}}\left(b_{1}, \ldots, b_{u+1}\right) \in\left\{b_{1}, b_{t}\right\}$ для любого $\tilde{b} \in O_{2}^{*} \cup \ldots \cup O_{\mu}^{*}$. Если $u+1 \leqslant[k / 2]+1$, то $\mu=1$ и перестановкой переменных можно получить $l_{u+1}$ из $g_{\tau_{1}}$. Предположим, что $u+1>[k / 2]+1, \mu \geqslant 2$ и в $\left[\left\{g_{1}\right\}\right]_{G^{*}}$ найдется $g_{\tau_{\gamma}}\left(x_{1}, \ldots, x_{u+1}\right)$ такая, что $\vec{B}_{u} g_{\tau_{\gamma}}=x_{1}, g_{\tau_{\gamma}}=x_{t}$ на $O_{1}^{*} \cup \ldots \cup O_{\gamma}^{*}, 1 \leqslant \gamma \leqslant \mu$. Если $g_{\tau_{\gamma}}\left(b_{1}^{1}, \ldots, b_{u+1}^{1}\right)=b_{t}^{1}$ для некоторого набора $\tilde{b}^{1}=\left(b_{1}^{1}, \ldots, b_{u+1}^{1}\right) \in O_{\gamma+1}^{*}$, то аналогично предыдущему рассуждению в $\left[\left\{g_{\tau_{\gamma}}\right\}\right]_{G^{*}}$ найдется $g_{\tau_{\gamma+1}}\left(x_{1}, \ldots, x_{u+1}\right)$ такая, что $\vec{B}_{u} g_{\tau_{\gamma+1}}=x_{1}, g_{\tau_{\gamma+1}}=x_{t}$ на $O_{1}^{*} \cup \ldots \cup O_{\gamma+1}^{*}$. В противном случае $g_{\tau_{\gamma}} \equiv x_{1}$ на $O_{\gamma+1}^{*}$. Поскольку степень транзитивности группы $G \geqslant[k / 2]+1$, согласно предложению 1 в $O_{\gamma+1}^{*}$ всегда найдется вектор $\tilde{a}^{1}=\left(a_{1}^{1}, \ldots, a_{u+1}^{1}\right)$ такой, что $a_{1}^{1}=a_{1}$, $a_{t}^{1}=a_{t}, M_{\tilde{a}^{1}}=M_{\tilde{a}}$. Пусть

$$
g_{\tau_{\gamma}+1}\left(x_{1}, \ldots, x_{u+1}\right)=g_{\tau_{\gamma}}\left(x_{1}, x_{\delta(2)}, \ldots, x_{\delta(t-1)}, x_{t}, x_{\delta(t+1)}, \ldots, x_{u+1}\right),
$$

где перестановка

$$
\left(\begin{array}{cccccccc}
x_{1}, & x_{2}, & \ldots, & x_{t-1}, & x_{t}, & x_{t+1}, & \ldots, & x_{u+1} \\
x_{1}, & x_{\delta(2)}, & \ldots, & x_{\delta(t-1)}, & x_{t}, & x_{\delta(t+1)}, & \ldots, & x_{\delta(u+1)}
\end{array}\right)
$$

такова, что

$$
\left(a_{1}^{1}, a_{\delta(2)}^{1}, \ldots, a_{\delta(t-1)}^{1}, a_{t}^{1}, a_{\delta(t+1)}^{1}, \ldots, a_{\delta(u+1)}^{1}\right)=\left(a_{1}, a_{2}, \ldots, a_{u+1}\right) .
$$


Пусть

$$
g_{\tau_{\gamma}+2}\left(x_{1}, \ldots, x_{u+1}\right)=g_{\tau_{\gamma}+1}\left(g_{\tau_{\gamma}}\left(x_{1}, \ldots, x_{u+1}\right), x_{2}, \ldots, x_{u+1}\right) .
$$

Тогда $\vec{B}_{u} g_{\tau_{\gamma}+2}=x_{1}, g_{\tau_{\gamma}+2}\left(b_{1}, \ldots, b_{u+1}\right)=b_{t}$ для любого $\tilde{b} \in I_{k, u+1}^{u+1}$ такого, что $g_{\tau_{\gamma}}(\tilde{b})=b_{t}$. Кроме того, $g_{\tau_{\gamma}+2}\left(a_{1}^{1}, \ldots, a_{u+1}^{1}\right)=a_{t}^{1}$. Возвращаемся к рассмотренной ситуации. Тем самым доказано, что $[\{g\}]_{G^{*}}$ содержит $g_{\tau_{\mu}}\left(x_{1}, \ldots, x_{u+1}\right)$ такую, что $\vec{B}_{u} g_{\tau_{\mu}}=x_{1}, I_{k, u+1} g_{\tau_{\mu}}=x_{t}$, т. е. $[\{g\}]_{G^{*}} \ni l_{u+1}$.

2. Пусть $g\left(e_{1}, \ldots, e_{u+1}\right)=e_{u+2} \in E_{k} \backslash M_{\tilde{e}}$ для некоторого $\tilde{e}=\left(e_{1}, \ldots, e_{u+1}\right) \in$ $I_{k, u+1}^{u+1}$. Без ограничения общности можно предположить, что $\tilde{e}=\tilde{a}, e_{u+2}=a_{u+2}$, $\left\{a_{1}, \ldots, a_{k}\right\}=E_{k}$. Построим последовательность формул $u^{0}, u^{1}, u^{2}, \ldots, u^{k}$ следующим образом. Пусть

$$
\begin{aligned}
u^{0} & =u^{0}[g]=g\left(x_{1}, \ldots, x_{u+1}\right), \\
u^{1} & =u^{1}\left[g, g_{1}^{1}, g_{2}^{1}\right]=g\left(x_{1}, \ldots, x_{u+1}, g_{1}^{1}\left(x_{1}, x_{i_{2}^{1,1}}, \ldots, x_{i_{u+1}^{1,1}}\right), g_{2}^{1}\left(x_{1}, x_{i_{2}^{1,2}}, \ldots, x_{i_{u+1}^{1,2}}\right),\right. \\
& \ldots \\
u^{r} & =u^{r}\left[g, g_{1}^{1}, g_{2}^{1}, \ldots, g_{1}^{r}, \ldots, g_{2^{r}}^{r}\right], \quad k \geqslant r \geqslant 1,
\end{aligned}
$$

получена из формулы $u^{r-1}\left[g, g_{1}^{1}, g_{2}^{1}, \ldots, g_{1}^{r-1}, \ldots, g_{2^{r-1}}^{r-1}\right]$ подстановкой функции $g_{\varkappa}^{r}\left(x, x_{i_{2}^{r, \varkappa}}, \ldots, x_{i_{u+1}^{r, \varkappa}}\right)$ из $[\{g\}]_{G^{*}}, \varkappa=1, \ldots, 2^{r}$, вместо аргументов $x_{i_{u}^{r-1,1}, x_{i_{u+1}^{r-1,1}}}$, $\ldots, x_{i_{u}^{r-1,2^{r-1}}}, x_{i_{u+1}^{r-1,2^{r-1}}} \quad$ функций $\quad g_{1}^{r-1}, \ldots, g_{2^{r-1}}^{r-1} \quad$ соответственно, $\quad$ где $\left\{x_{i_{2}^{r, \varkappa}}, \ldots, x_{i_{u+1}^{r, \varkappa}}\right\}^{+1}-u$-элементные подмножества множества $X_{k}=\left\{x_{1}, \ldots, x_{k}\right\}$, $\vec{B}_{u} g_{\varkappa}^{r}=x_{1}$,

$$
g_{\varkappa}^{r}\left(a_{1}, a_{i_{2}^{r, \varkappa}}, \ldots, a_{i_{u+1}^{r, \varkappa}}\right)=a_{i_{u+1}^{r-1,(\varkappa+1) / 2}} \in E_{k} \backslash\left\{a_{1}, a_{i_{2}^{r, \varkappa}}, \ldots, a_{i_{u+1}^{r, \varkappa}}\right\}
$$

при нечетном $\varkappa$,

$$
\vec{B}_{u} g_{\varkappa}^{r}=x_{1}, \quad g_{\varkappa}^{r}\left(a_{1}, a_{i_{2}^{r, \varkappa}}, \ldots, a_{i_{u+1}^{r, \varkappa}}\right)=a_{i_{u+1}^{r-1, \varkappa / 2}} \in E_{k} \backslash\left\{a_{1}, a_{i_{2}^{r, \varkappa}}, \ldots, a_{i_{u+1}^{r, \varkappa}}\right\}
$$

при четном $\varkappa, \varkappa=1, \ldots, 2^{r}$ (такое построение возможно, согласно предложению 1). С помощью формулы $u^{k}$ построим формулы $u_{0}^{*}, u_{1}^{*}, \ldots, u_{q}^{*}, q=\left(\begin{array}{c}k-1 \\ 2\end{array}\right)$ следующим индуктивным образом. Пусть $\left\{\left(i_{1}, j_{2}\right), \ldots,\left(i_{q}, j_{q}\right)\right\}$ - множество всех пар различных чисел из $\{2, \ldots, k\} \times\{2, \ldots, k\}$. Пусть $u_{0}^{*}=u^{k}$. Предположим, что $u_{0}^{*}, \ldots, u_{d}^{*}, 0 \leqslant d<q$, построены. Опишем способ построения $u_{d+1}^{*}$. Если $\left\{x_{i_{d+1}}, x_{j_{d+1}}\right\} \subset X_{g_{d+1}^{k}}\left(X_{g}\right.$ - список переменных функции $\left.g\right)$, то $u_{d+1}^{*}=u_{d}^{*}$. В противном случае существует $x_{t} \in X_{g_{d+1}^{k}} \backslash\left\{x_{i_{d+1}}, x_{j_{d+1}}, x_{1}\right\}$. Найдется $g_{d+1}^{k+1}\left(x_{1}, x_{i_{2}^{k+1, d+1}}, \ldots, x_{i_{u+1}^{k+1, d+1}}\right) \in G^{*}(g)$ такая, что $\vec{B}_{u} g_{d+1}^{k+1}=x_{1}$,

$$
\begin{gathered}
g_{d+1}^{k+1}\left(a_{1}, a_{i_{2}^{k+1, d+1}}, \ldots, a_{i_{u+1}^{k+1, d+1}}\right)=a_{t} \in E_{k} \backslash\left\{a_{1}, a_{i_{2}^{k+1, d+1}}, \ldots, a_{i_{u+1}^{k+1, d+1}}\right\} \\
\left\{i_{d+1}, j_{d+1}\right\} \subset\left\{i_{2}^{k+1, d+1}, \ldots, i_{u+1}^{k+1, d+1}\right\} .
\end{gathered}
$$

Тогда формула $u_{d+1}^{*}$ получена из формулы $u_{d}^{*}$ подстановкой функции $g_{d+1}^{k+1}\left(x_{1}, x_{i_{2}^{k+1, d+1}}, \ldots, x_{i_{u+1}^{k+1, d+1}}\right)$ вместо аргумента $x_{t}$ функции $g_{d+1}^{r}$ формулы $u_{d}^{*}$. Нетрудно видеть, что $u_{q}^{*}$ реализует функцию $g^{*}\left(x_{1}, \ldots, x_{k}\right)$ такую, что $\vec{B}_{k-1} g^{*}=x_{1}$, $g^{*}\left(a_{1}, \ldots, a_{k}\right) \neq a_{1}$. Рассуждая аналогично подслучаю 1 , находим, что $l_{k} \in$ 
$\left[\left\{g^{*}\right\}\right]_{G^{*}}$. Для любого $r, 3 \leqslant r \leqslant k-u$, согласно предложению 1 найдется $\varphi_{r}$ из $G$ такая, что $\varphi_{r}\left(a_{1}\right)=a_{1}, \varphi_{r}\left(a_{u+r}\right)=a_{u+2},\left\{\varphi_{r}^{-1}\left(a_{2}\right), \ldots, \varphi_{r}^{-1}\left(a_{u+1}\right)\right\}=\left\{a_{2}, \ldots, a_{u+1}\right\}$. Тогда $\left\langle\varphi_{r}\right\rangle\left(g\left(\varphi_{r}^{-1}\left(a_{1}\right), \ldots, \varphi_{r}^{-1}\left(a_{u+1}\right)\right)\right)=a_{u+r}$. Легко видеть, что

$$
\left(\varphi_{r}^{-1}\left(a_{1}\right), \ldots, \varphi_{r}^{-1}\left(a_{u+1}\right)\right)=\left(a_{1}, a_{\delta_{r}(2)}, \ldots, a_{\delta_{r}(u+1)}\right),
$$

где $\delta_{r}$ - некоторая перестановка, действующая на $\{2, \ldots, u+1\}$. Пусть

$$
g_{u+r}^{*}\left(x_{1}, \ldots, x_{u}\right)=\left\langle\varphi_{r}\right\rangle\left(g\left(a_{1}, a_{\delta_{r}(2)}, \ldots, a_{\delta_{r}(u+1)}\right) .\right.
$$

Ясно, что $\vec{B}_{u} g_{u+r}^{*}=x_{1}, g_{u+r}^{*}\left(a_{1}, \ldots, a_{u+1}\right)=a_{u+r}$. Пусть

$$
\begin{aligned}
& g_{0}^{*}\left(x_{1}, \ldots, x_{u+1}\right)=l_{k}\left(x_{1}, \ldots, x_{u}, g\left(x_{1}, \ldots, x_{u+1}\right),\right. \\
& \left.\quad g_{u+3}^{*}\left(x_{1}, \ldots, x_{u+1}\right), \ldots, g_{k}^{*}\left(x_{1}, \ldots, x_{u+1}\right), x_{u+1}\right) .
\end{aligned}
$$

Тогда $\vec{B}_{u} g_{0}^{*}=x_{1}, g_{0}^{*}\left(b_{1}, \ldots, b_{u+1}\right) \in\left\{b_{1}, b_{u+1}\right\}$ для любого $\tilde{b}=\left(b_{1}, \ldots, b_{u+1}\right) \in$ $I_{k, u+1}^{u+1}$. Кроме того, $g_{0}^{*}\left(a_{1}, \ldots, a_{u+1}\right)=a_{u+1}$. Возвращаемся к рассмотренному подслучаю 1 , т.е. можно получить $l_{u+1}$ из $[\{f\}]_{G^{*}}$. Тогда согласно предложению $2 \mathrm{ORD}[\{f\}]_{G^{*}} \leqslant k$.

Случай II. $f\left(x_{1}, \ldots, x_{n}\right) \in P_{k}^{2} \vec{C}_{4} \backslash P_{k}^{2} O_{1}$.

1. Пусть $f\left(x_{1}, \ldots, x_{n}\right) \in M_{k}$, где $M(k)=P_{k}^{2} \vec{O}_{1} \backslash P_{k}^{2} O_{1}$ при $k \geqslant 3, k \neq 4$ и $M(4)=P_{4}^{2} \vec{O}_{1} \backslash P_{4}^{2} \tilde{O}_{1}$. Подстановкой переменных $x, y$ из функции $f$ можно получить $f_{1}(x, y) \in M(k)$. Без ограничения общности можно предполагать, что $B_{\{0,1\}} f_{1}(x, y)=x$ и $f_{1}\left(a_{1}, b_{1}\right)=b_{1}$ для некоторой пары $\left(a_{1}, b_{1}\right) \in E_{k}^{2}$. Предположим, что $f_{1}\left(a_{1}, b_{i}\right)=b_{i}, f_{1}\left(a_{1}, b_{j}\right)=a_{1}$, где $i=1, \ldots, q, j=q+1, \ldots, k-1$. Если $k \geqslant 5$, то в $G$ найдется

$$
\pi_{1}=\left(b_{q+1} b_{q} \ldots\right)(01)\left(a_{1}\right)(\ldots) \ldots(\ldots) .
$$

Пусть $f_{1}^{1}(x, y)=f_{1}\left(f_{1}^{\pi_{1}}(x, y), y\right)$. Легко видеть, что $B_{\{0,1\}} f_{1}^{1}(x, y)=x, f_{1}^{1}(a, b)=b$ на таких наборах $(a, b)$, что $f_{1}(a, b)=b$. Кроме того $f_{1}^{1}\left(a_{1}, b_{q+1}\right)=b_{q+1}$. Таким образом, из $\left[\left\{f_{1}\right\}\right]_{G^{*}}$ можно получить $f_{1,1}$ такую, что $B_{\{0,1\}} f_{1,1}(x, y)=x$, $f_{1,1}\left(a_{1}, b\right)=b$ для любого $b \in E_{k}$. Предположим, что $f_{1,1}\left(a_{2}, b_{t}\right)=a_{2}$ для некоторого $b_{t} \in E_{k}$. В $G$ найдется

$$
\pi_{2}=(01)\left(b_{t}\right)\left(a_{2} a_{1} \ldots\right)(\ldots) \ldots(\ldots) .
$$

Пусть $f_{1,2}(x, y)=f_{1,1}\left(f_{1,1}^{\pi_{2}}(x, y), y\right)$. Ясно, что $B_{\{0,1\}} f_{1,2}(x, y)=x, f_{1,2}(a, b)=b$ на таких наборах $(a, b)$, что $f_{1,1}(a, b)=b$. Кроме того, $f_{1,2}\left(a_{2}, b_{t}\right)=b_{t}$. Продолжая эту процедуру, из $f_{1} G^{*}$-замыканием можно получить $f^{\overrightarrow{0}}(x, y)$.

Очевидной модификацией этой схемы можно получить аналогичный результат при $k=3,4$. Пусть $f_{2}(x, y, z)=f^{\overrightarrow{0}}\left(f^{\overrightarrow{0}}(x, y), f^{\overrightarrow{0}}(z, x)\right)$. Легко проверить, что $\vec{B}_{2} f_{2}(x, y, z)=x, f_{2}(0,2,1)=1$. Из случая I следует, что $\left[\left\{f_{1}\right\}\right]_{G^{*}} \supseteq\left[\left\{f_{2}\right\}\right]_{G^{*}} \ni l_{3}$.

2. Пусть $f\left(x_{1}, \ldots, x_{n}\right) \in P_{4}^{2} \tilde{O}_{1} \backslash P_{4}^{2} O_{1}$ при $k=4$. Аналогично подслучаю 1 , легко доказать, что $f^{\tilde{0}}(x, y) \in[\{f\}]_{G^{*}}$. Пусть $\tilde{f}_{1}(x, y, z)=f^{\overline{0}}\left(f^{\overline{0}}\left(f^{\tilde{0}}(x, y), f^{\tilde{0}}(z, x)\right), z\right)$. Легко проверить, что $\tilde{f}_{1}(x)=x$ на $\{0,1\}^{3} \cup\{2,3\}^{3}$ и $f_{1}=z$ на остальных наборах. Пусть $\delta_{1}=(0231), \tilde{f}_{2}=\left\langle\delta_{1}\right\rangle\left(\tilde{f}_{1}\right), \tilde{f}_{3}(x, y, z)=f^{\tilde{0}}\left(f_{1}(x, y, z), f_{z}(x, y, z)\right)$, $\delta_{2}=(0123), \tilde{f}_{4}=\left\langle\delta_{2}\right\rangle\left(\tilde{f}_{3}\right)$. Тогда нетрудно проверить, что $l_{3}(x, y, z)=f^{5}(z, y, x)$, где $f^{5}(x, y, z)=f^{\tilde{0}}\left(\tilde{f}_{3}(x, y, z), \tilde{f}_{4}(x, y, z)\right)$. 
3. Пусть $f \in P_{k}^{2} \vec{C}_{4} \backslash P_{k}^{2} \vec{O}_{1}$. Без ограничения общности можно предполагать, что $B_{\{0,1\}} f \in C_{4} \backslash O_{1}$. Согласно предложению $\mathrm{P}[10] B_{\{0,1\}}[\{f\}]_{G^{*}}$ либо содержит класс $D_{2}$, либо совпадает с классом $L_{4}$.

(a) Если $D_{2} \subseteq B_{\{0,1\}}[\{f\}]_{G^{*}}$, то в $[\{f\}]_{G^{*}}$ найдется $f_{3}(x, y, z)$ такая, что $B_{\{0,1\}} f_{3}(x, y, z)=B_{\{0,1\}} d(x, y, z)$. Если $\vec{B}_{2} f_{3}=\vec{B}_{2} d$, то $f_{3}$ - мажоритарная функция. Согласно доказательству леммы 4, работы [9] $\mathrm{ORD}[\{f\}]_{G^{*}} \leqslant k^{3}$. Если $f_{3}(a, a, b)=b$ для некоторых различных $a, b \in E_{k}$, то подстановкой переменных из $f_{3}$ можно получить $f_{3}^{1}(x, y)$ такую, что $B_{\{0,1\}} f_{3}^{1}(x, y)=x, f_{3}^{1}(a, b)=b$. Очевидной модификацией схемы, изложенной в пункте 1 , можно получить $f_{3}^{2}(x, y) \in\left[\left\{f_{3}^{1}\right\}\right]_{G \text { * }}$ такую, что $f_{3}^{2}(x, y) \in P_{k}^{2} \vec{O}_{1} \backslash P_{k}^{2} O_{1}$. Согласно пунктам $1,2\left[\left\{f_{3}^{2}\right\}\right]_{G^{*}}$ содержит либо $\left\{f^{0}, l_{3}\right\}$ для любого $k \geqslant 3$, либо $\left\{f^{\overrightarrow{0}}, l_{3}\right\}$ для $k=4$.

(b) Если $L_{4}=B_{\{0,1\}}[\{f\}]_{G^{*}}$, то в $[\{f\}]_{G^{*}}$ найдется $f_{4}(x, y, z)$ такая, что $B_{\{0,1\}} f_{4}(x, y, z)=x \oplus y \oplus z=B_{\{0,1\}} s(x, y, z)$.

$(\alpha)$ Если $\vec{B}_{2}[\{f\}]_{G^{*}}=\vec{B}_{2} S_{k} L_{k}^{*}$, то $\vec{B}_{2} f_{4}=\vec{B}_{2} s$.

Если $f_{4}(x, y, z)=s(x, y, z)$ с точностью до перестановки переменных, то $\left[\left\{f_{4}\right\}\right]_{G^{*}}=S_{k} L_{4}^{*} \ni l_{3}(x, y, z)$. Легко видеть, что $\vec{B}_{2}\left[\left\{f_{4}\right\}\right]_{G^{*}}=\vec{B}_{2}[\{f\}]_{G^{*}}$. Отсода и из доказательства леммы 2 работы [9] следует, что из функций $f_{4}, l_{3}$ и всех таких функций $f^{\varepsilon}\left(x_{1}, \ldots, x_{n}\right), \varepsilon=\Delta_{n}^{3} \cup \ldots \cup \Delta_{n}^{k}$, которые существенно зависят не более, чем от $k$ переменных, суперпозициями можно построить $f$, т. e. $\operatorname{ORD}[\{f\}]_{G^{*}} \leqslant k$.

Пусть $f_{4}\left(a_{1}, a_{2}, a_{3}\right)=a_{i}, f_{4}\left(b_{1}, b_{2}, b_{3}\right)=b_{j}$ для некоторых 3-элементных наборов $\tilde{a}=\left(a_{1}, a_{2}, a_{3}\right), \tilde{b}=\left(b_{1}, b_{2}, b_{3}\right)$, где $\tilde{a} \neq \tilde{b}, i \neq j, i \in\{1,2,3\}$. Если $j \in\{1,2,3\}$, то без ограничения общности можно считать, что $i=1, j=3$. Пусть $f_{4,1}(x, y, z)=$ $f_{4}\left(f_{4}(x, y, z), y, z\right)$. Тогда $\vec{B}_{2} f_{4,1}=x, f_{4,1}\left(b_{1}, b_{2}, b_{3}\right)=b_{2}$. Согласно рассмотренному случаю 1 справедливо включение $l_{3} \in\left[\left\{f_{4,1}\right\}\right]_{G^{*}}$. Возвращаемся к предыдущей ситуации. Если $j=4$ и $b_{4} \notin\left\{b_{1}, b_{2}, b_{3}\right\}$, то в $\left[\left\{f_{4}\right\}\right]_{G^{*}}$ найдется $f_{4,2}(x, y, z)$ такая, что $\vec{B}_{2} f_{4,2}=\vec{B}_{2} s, f_{4,2}\left(b_{4}, b_{2}, b_{3}\right)=b_{2}$. Пусть $f_{4,3}(x, y, z)=f_{4,2}\left(f_{4}(x, y, z), y, z\right)$. Тогда $\vec{B}_{2} f_{4,3} \equiv x, f_{4,3}\left(b_{1}, b_{2}, b_{3}\right)=b_{2}$. Приходим к предыдущей ситуации.

Пусть $f_{4}\left(a_{1}, a_{2}, a_{3}\right)=a_{4} \notin\left\{a_{1}, a_{2}, a_{3}\right\}$ для произвольного 3-элементного набора $\left(a_{1}, a_{2}, a_{3}\right)$. Если $k \geqslant 5$, то в $\left[\left\{f_{4}\right\}\right]_{G^{*}}$ найдется $f_{4,4}(x, y, z)$ такая, что $\vec{B}_{2} f_{4,4}=$ $\vec{B}_{2} s, f_{4,4}\left(a_{4}, a_{2}, a_{3}\right)=a_{5} \notin\left\{a_{1}, a_{2}, a_{3}, a_{4}\right\}$. Пусть $f_{4,5}(x, y, z)=f_{4,4}\left(f_{4}(x, y, z), y, z\right)$. Tогда $\vec{B}_{2} f_{4,5}=x, f_{4,5}\left(a_{1}, a_{2}, a_{3}\right)=a_{5}$. Приходим к рассмотренной ситуации. При $k=4$ имеем $f_{4}(x, y, z)=f_{0}(x, y, z)$. Если $\left[\left\{f_{0}\right\}\right]_{G^{*}}=[\{f\}]_{G^{*}}$, то ORD $[\{f\}]_{G^{*}} \leqslant 3$. В противном случае, согласно определению 3.к. $S_{4}^{4} L_{4}$ (см. [11]), подстановкой переменных и функции $f_{0}$ из функции $f$ можно получить $f_{4,6}(x, y, z) \notin S_{4}^{4} L_{4}$. Легко видеть, что $f_{4,6}$ удовлетворяет условиям рассмотренных ситуаций.

$(\beta)$ Если $\vec{B}_{2}[\{f\}]_{G^{*}} \neq \vec{B}_{2} S_{k} L_{4}^{*}$, то из $f$ подстановкой переменных $x, y$ некоторой модификацией схемы из пункта 1 можно получить $f_{5}(x, y) \in P_{k}^{2} \vec{O}_{1} \backslash P_{k}^{2} O_{1}$. Возвращаемся к рассмотренной ситуации.

Итак в случае II нами доказано, что либо $\mathrm{ORD}[\{f\}]_{G^{*}} \leqslant k^{3}$, либо $[\{f\}]_{G^{*}}$ содержит $\left\{f^{\overrightarrow{0}}, l_{3}\right\}$, либо $[\{f\}]_{G^{*}}$ содержит $\left\{f^{\tilde{0}}, l_{3}\right\}$ при $k=4$.

Предположим, что $[\{f\}]_{G^{*}} \supset\left\{f^{\overrightarrow{0}}, l_{3}\right\}$. Согласно предложению $\mathrm{P}[10]$ и результатам работы [1] всегда существуют $\tilde{f}^{1}(x, y, z), \ldots, \tilde{f}^{s_{1}}(x, y, z)\left(s_{1}=k(k-1) / 2\right)$ из $[\{f\}]_{G^{*}}$ такие, что $B_{I_{t}^{2}} \tilde{f}^{t}\left(x_{1}, \ldots, x_{n}\right)=B_{I_{t}^{2}} f\left(x_{1}, \ldots, x_{n}\right), t=1, \ldots, s_{1}$. Используем индукцию и предположим, что $\left[\left\{f^{\vec{c}}, \tilde{f}^{1}, \ldots, \tilde{f}^{q}\right\}\right]_{G^{*}}$ содержит $h^{q}\left(x_{1}, \ldots, x_{n}\right)$ такую, что $B_{I_{t}^{2}} h^{q} \equiv B_{I_{t}^{2}} f, t=1, \ldots, q, 1 \leqslant q<s_{1}$. В $\left[\left\{f^{\vec{\theta}}\right\}\right]_{G^{*}}$ найдется $f_{q+1}^{\overrightarrow{0}}(x, y)$ такая, 
что $f_{q+1}^{\overrightarrow{0}}(x, y)=y$ на $B_{I_{q+1}^{2}}$ и $f_{q+1}^{\overrightarrow{0}}(x, y)=x$ на остальных наборах. Пусть

$$
h^{q+1}\left(x_{1}, \ldots, x_{n}\right)=f_{q+1}^{\overrightarrow{0}}\left(h^{q}\left(x_{1}, \ldots, x_{n}\right), \tilde{f}^{q+1}\left(x_{1}, \ldots, x_{n}\right)\right) .
$$

Очевидно, что $B_{I_{t}^{2}} h^{q+1}=B_{I_{t}^{2}} f, t=1, \ldots, q+1$. Тем самым доказано, что $\left[\left\{f^{\overrightarrow{0}}, \tilde{f}^{1}, \ldots, \tilde{f}^{s_{1}}\right\}\right]_{G^{*}}$ содержит $h^{s_{1}}\left(x_{1}, \ldots, x_{n}\right)$ такую, что $\vec{B}_{2} h^{s_{1}}=\vec{B}_{2} f$. По аналогичной схеме такой же результат получается при $[\{f\}]_{G^{*}} \supset\left\{f^{\overrightarrow{0}}, l_{3}\right\}(k=4)$. Из $h^{s_{1}}, l_{3}$ и всех таких $f^{\varepsilon}\left(x_{1}, \ldots, x_{n}\right), \varepsilon=\Delta_{n}^{3} \cup \ldots \cup \Delta_{n}^{q}$, которые зависят существенно не более, чем от $k$ переменных, суперпозициями можно получить функцию $f$, т. е. ORD $[\{f\}]_{G^{*}} \leqslant k$.

Случай III. $f\left(x_{1}, \ldots, x_{n}\right) \in P_{k}^{\alpha} \backslash P_{k}^{2} \vec{C}_{4}$. Рассмотрим следующие всевозможные подслучаи, где $k=4$ в подслучаях 1-3 и $k=3$ в подслучае 4 и $k \geqslant 3$ в подслучае 5.

1. $f \in A_{4}^{4} L_{4}$. Согласно работе $[11], \operatorname{ORD}[\{f\}]_{G^{*}} \leqslant 4$.

2. $f \in V Q L_{4}^{\alpha} \backslash A_{4}^{4} L_{4}$. Тогда

$$
f\left(x_{1}, \ldots, x_{n}\right)=a_{1} x_{1}^{2}+\ldots \dot{+} a_{n} x_{n}^{2}+b_{1} x_{1} \dot{+} \ldots \dot{+} b_{n} x_{n},
$$

где $a_{i} \neq 0$ для некоторого $i, 1 \leqslant i \leqslant n, a_{1}+\ldots \dot{+} a_{n}=0, b_{1}+\ldots \dot{+} b_{n}=1$. Покажем, что $q^{V}(x, y)=x^{2} \dot{+} y^{2} \dot{+} \in[\{f\}]_{G^{*}}$. Подстановкой переменных из $f$ можно получить

$$
g\left(x_{1}, x_{2}\right)=a x_{1}^{2} \dot{+} a x_{2}^{2} \dot{+} c_{1} x_{1} \dot{+} c_{2} x_{2},
$$

где $a \neq 0, c_{1} \dot{+} c_{2}=1$. Пусть $\sigma_{1}=2 x, \sigma_{2}=3 x$. Если $a=2, a=3$, то соответственно

$$
g_{1}\left(x_{1}, x_{2}\right)=g^{\sigma_{2}}\left(x_{1}, x_{2}\right)=g^{\sigma_{1}}\left(x_{1}, x_{2}\right)=x_{1}^{2} \dot{+} a x_{2}^{2} \dot{+} c_{1} x_{1} \dot{+} c_{2} x_{2} .
$$

Если $\left(c_{1}, c_{2}\right) \in\{(0,1),(1,0)\}$, то перестановкой переменных из $g_{1}$ можно получить $g^{v}\left(x_{1}, x_{2}\right)$. Из оставшихся случаев, когда $\left(c_{1}, c_{2}\right) \in\{(2,3),(3,2)\}$ достаточно рассмотреть случаи, когда $\left(c_{1}, c_{2}\right) \in(2,3)$. Тогда

$$
\begin{aligned}
g_{2}\left(x_{1}, x_{2}\right) & =g_{1}^{\sigma_{1}}\left(x_{1}, x_{2}\right)=2 x_{1}^{2}+2 x_{2}^{2}+2 x_{1}+3 x_{2}, \\
g_{3}\left(x_{1}, x_{2}\right) & =g_{1}^{\sigma_{1}}\left(x_{1}, x_{2}\right)=3 x_{1}^{2}+3 x_{2}^{2}+2 x_{1}+3 x_{2}, \\
g_{4}\left(x_{1}, x_{2}\right) & =g_{1}\left(g_{3}\left(x_{1}, x_{2}\right), g_{2}\left(x_{1}, x_{2}\right)\right)=3 x_{1}^{2}+2 x_{2}^{2}=\gamma\left(x_{1}, x_{2}\right), \\
g_{5}\left(x_{1}, x_{2}, x_{3}\right) & =x_{1}+x_{2}+x_{3} \in\left[\left\{\gamma_{4}\right\}\right]_{G^{*}}=A_{4}^{4} L_{4}, \\
g_{6}\left(x_{1}, x_{2}\right) & =g_{5}\left(g_{1}\left(x_{1}, x_{2}\right), g_{4}\left(x_{1}, x_{2}\right), x_{2}\right)=q^{v}\left(x_{1}, x_{2}\right) .
\end{aligned}
$$

Покажем, что $\left[\left\{q^{v}\right\}\right]_{G^{*}} \ni f$. Пусть

$$
q_{1}^{v}(x, y)=\left\langle\sigma_{1}\right\rangle\left(q^{v}(x, y)\right)=2 x^{2}+2 y^{2}+x .
$$

Тогда

$$
q^{v}\left(q^{v}(x, y), q_{1}^{v}(y, x)\right)=3 x+2 y=\gamma_{4}(x, y) .
$$

Согласно работе [11]

$$
\left[\left\{\gamma_{4}\right\}\right]_{G^{*}} \equiv A_{4}^{4} L_{4} \ni f_{0}(x, y, z)=x+y+z .
$$

Отсюда следует, что

$$
\left.q_{2}^{v}(x, y, z)=f_{0}\left(q^{v}(x, y), x, z\right)\right)=x^{2} \dot{+} y^{2} \dot{+} z .
$$


Нетрудно проверить, что

$$
\begin{aligned}
\left\langle\sigma_{1}\right\rangle\left(q_{2}^{v}(x, y, z)\right) & =2 x^{2}+2 y^{2}+z, \\
\left\langle\sigma_{2}\right\rangle\left(q_{2}^{v}(x, y, z)\right) & =3 x^{2}+3 y^{2}+z, \\
q_{2}\left(x, y, 3 y^{2}+3 z^{2}+u\right) & =x^{2}+2 y^{2}+3 z^{2}+u
\end{aligned}
$$

Легко проверить, что из функций

$$
3 x+2 y, \quad x^{2}+y^{2}+z, \quad 2 x^{2}+2 y^{2}+z, \quad 3 x^{2}+3 y^{2}+z, \quad x^{2}+2 y^{2}+3 z^{2}+u
$$

суперпозициями можно построить все трехместные функции из $V Q L_{4}^{\alpha}$. Пусть $n>3$. Предположим, что среди коэффициентов $b_{1}, \ldots, b_{n}$ есть два одинаковых, например, $b_{1}=b_{2}$. Тогда

$$
f\left(x_{1}, \ldots, x_{n}\right)=a_{2} x_{1}^{2} \dot{+} a_{2} x_{2} \dot{+} b_{1} x_{1} \dot{+} b_{1} x_{2} \dot{+} f\left(x_{1}, x_{1}, x_{3}, \ldots, x_{n}\right) .
$$

Пусть все три числа $b_{1}, b_{2}, b_{3}$ различны. Так как в поле GF(4) $1+2+3=0$, из равенства

$$
b_{1} \dot{+} \ldots \dot{+} b_{n}=1 \text {, }
$$

следует, что среди коэффициентов $b_{1}, \ldots, b_{n}$ есть хотя бы один ненулевой. Тогда этот коэффициент совпадает с одним из коэффициентов $b_{1}, b_{2}, b_{3}$ и мы вновь можем выбрать тройку ненулевых коэффициентов, среди которых имеются два одинаковых. Итак, этой индуктивной схемой доказано, что $f \in\left[\left\{q^{v}\right\}\right]_{G^{*}}$, т. е. $\mathrm{ORD}[\{f\}]_{G^{*}}=2$.

3. $f \in V_{4}^{\alpha}$. Если $f \in V_{4}^{2} O_{1}$, то возвращаемся к случаю I. Если $f \in V_{4}^{2} \tilde{D}_{1} \backslash V_{4}^{2} O_{1}$, то аналогично подслучаям 2,3 случая II находим, что $\mathrm{ORD}\left([\{f\}]_{G^{*}}\right) \leqslant 4^{3}$.

Если $f \in V_{4}^{\alpha} \backslash\left(V_{4}^{2} \tilde{D}_{1} \cup V Q L_{4}^{\alpha}\right)$, то подстановкой переменных из $f$ можно получить $g_{1}(x, y) \in V_{4}^{\alpha} \backslash V_{4}^{2} \tilde{D}_{1}$. Покажем, что $A_{4}^{4} L_{4} \subseteq\left[\left\{g_{1}\right\}\right]_{G^{*}}$. Рассмотрим всевозможные ситуации.

(a) Пусть

$$
g_{1}(x, y)= \begin{cases}v, v \in\{z, u\}, & \text { если }(x, y, z, u) \in V_{i}^{1}, i=1,2,3 \\ x, & \text { если } x=y\end{cases}
$$

Тогда $g_{1}$ с точностью до перестановки переменных совпадает с $\gamma_{4}$.

(b) Для некоторых $i, j, t,\{i, j, t\}=\{1,2,3\}$ пусть

$$
g_{1}(x, y)= \begin{cases}v, v \in\{z, u\}, & \text { если }\{x, y, z, u\} \in V_{i}^{1} \cup V_{j}^{1}, \\ v^{\prime}, v^{\prime} \in\{z, u\} \backslash\{v\}, & \text { если }\{x, y, z, u\} \in V_{t}, \\ x, & \text { если } x=y\end{cases}
$$

Благодаря отношению $G^{*}$ и перестановке переменных достаточно рассмотреть ситуацию, когда $i=1, j=2, t=3, v=u, v^{\prime}=z$. Пусть $\varphi_{1}=(032)(1)$, $\varphi_{2}=(023)(1)$. Легко проверить, что

$$
h^{1}(x, y)=g^{\varphi_{1}}\left(g^{\varphi_{2}}(x, y), g_{1}(x, y)\right)=x^{2}+y^{2} \dot{+} 3 x+2 y .
$$

Согласно предыдущему подслучаю 2 имеем $\gamma_{4} \in V Q L_{4}^{\alpha}=\left[\left\{h_{1}^{1}\right\}\right]_{G^{*}}$. 
(с) Для некоторых $i, j, t,\{i, j, t\}=\{1,2,3\}$ пусть

$$
g_{1}(x, y)= \begin{cases}v, v \in\{z, u\}, & \text { если }\{x, y, z, u\} \in V_{i}^{1} \cup V_{j}^{1}, \\ v^{\prime}, v^{\prime} \in\{x, y\}, & \text { если }\{x, y, z, u\} \in V_{t}^{1} \\ x, & \text { если } x=y\end{cases}
$$

Пусть $i=1, j=2, t=3$. Если $v=u, v^{\prime}=x$, то

$$
h_{1}^{2}(x, y)=g^{\varphi_{1}}\left(g_{1}(x, y), y\right)= \begin{cases}v, & \text { если }(x, y, z, u) \in V_{2}^{1} \cup V_{3}^{1}, \\ z, & \text { если }(x, y, z, u) \in V_{1}^{1} \\ x, & \text { если } x=y .\end{cases}
$$

Возвращаемся к ситуации (b). Если $v=u, v^{\prime}=y$, то

$$
h_{1}^{3}(x, y)=g^{\varphi_{1}}\left(x, g_{1}(x, y)\right)= \begin{cases}z, & \text { если }(x, y, z, u) \in V_{1}^{1} \cup V_{2}^{1}, \\ u, & \text { если }(x, y, z, u) \in V_{3}^{1} \\ x, & \text { если } x=y .\end{cases}
$$

Снова возвращаемся к ситуации (b). Остальные варианты этого случая сводятся к рассмотренным благодаря $G^{*}$ и перестановке переменных.

(d) Для некоторого $i, 1 \leqslant i \leqslant 3$ пусть

$$
g_{1}(x, y)= \begin{cases}v, v \in\{z, u\}, & \text { если }(x, y, z, u) \in V_{i}^{1}, \\ v^{\prime}, v^{\prime} \in\{x, y\} & \text { в остальных случаях. }\end{cases}
$$

Достаточно рассмотреть варианты, когда $v=u, v^{\prime} \in\{x, y\}$. При $v^{\prime}=x$

$$
h_{1}^{2}(x, y)= \begin{cases}u, & \text { если }(x, y, z, u) \in V_{1}^{1} \cup V_{2}^{1}, \\ x & \text { в остальных случаях. }\end{cases}
$$

При $v^{\prime}=y$

$$
h_{1}^{4}(x, y)=g_{1}^{\varphi_{2}}\left(x, g_{1}(x, y)\right)= \begin{cases}u, & \text { если }(x, y, z, u) \in V_{1}^{1} \cup V_{3}^{1} \\ x & \text { в остальных случаях }\end{cases}
$$

В обоих вариантах приходим к ситуации (c).

(е) Для некоторых $i, j, t,\{i, j, t\}=\{1,2,3\}$,

$$
g_{1}(x, y)= \begin{cases}u, & \text { если }(x, y, z, u) \in V_{i}^{1}, \\ z, & \text { если }(x, y, z, u) \in V_{j}^{1}, \\ v, v \in\{x, y\}, & \text { если }(x, y, z, u) \in V_{t}^{1} .\end{cases}
$$

При $v=y,(i, j, t)=(1,2,3)((i, j, t)=(3,1,2),(i, j, t)=(2,3,1))$ имеем

$$
g_{1}(x, y)=3 x^{2}+3 y^{2}+x \quad\left(g_{1}(x, y)=2 x^{2}+2 y^{2}+x, \quad g_{1}(x, y)=x^{2}+y^{2}+x\right) .
$$

Согласно подслучаю $2 \gamma_{4} \in\left[\left\{g_{1}\right\}\right]_{G^{*}}=V Q L_{4}^{\alpha}$. При $v=y,(i, j, t)=(2,1,3)$

$$
h_{1}^{5}(x, y)=g_{1}\left(g^{\varphi_{1}}(x, y), y\right)= \begin{cases}z, & \text { если }(x, y, z, u) \in V_{3}^{1}, \\ y & \text { в остальных случаях }\end{cases}
$$


Возвращаемся к ситуации (d). Остальные варианты сводятся к рассмотренным благодаря $G^{*}$ и перестановке переменных.

(g) Для некоторых $i, j, 1 \leqslant i \neq j \leqslant 3$,

$$
g_{1}(x, y)= \begin{cases}v, v \in\{u, z\}, & \text { если }(x, y, z, u) \in V_{i}^{1}, \\ x, & \text { если }(x, y, z, u) \in V_{j}^{1}, \\ y & \text { в остальных случаях }\end{cases}
$$

При $v=u,(i, j)=(1,3),((i, j)=(3,2),(i, j)=(2,1))$ соответственно

$g_{1}(x, y)=x^{2} \dot{+} y^{2} \dot{+} 3 x+2 y, \quad g_{1}(x, y)=3 x^{2} \dot{+} 3 y^{2} \dot{+} 3 x+2 y, \quad g_{1}(x, y)=2 x^{2} \dot{+} 2 y^{2} \dot{+} 3 x \dot{+} 2 y$.

При $v=u,(i, j)=(1,2)$

$$
h_{1}^{2}(x, y)= \begin{cases}u, & \text { если }(x, y, z, u) \in V_{1}^{1} \cup V_{2}^{1}, \\ y & \text { в остальных случаях }\end{cases}
$$

Приходим к ситуации (с). Остальные варианты сводятся к рассмотренным благодаря $G^{*}$ и перестановке переменных.

Итак, доказано, что $\left[\left\{g_{1}\right\}\right]_{G^{*}} \supset\left[\left\{\gamma_{4}\right\}\right]_{G^{*}}=A_{4}^{4} L_{4}$.

Обычным образом определим $U(R)$ - класс всех $k$-значных функций, coхраняющих отношение $R$.

Рассуждая аналогично доказательству леммы 29 работы [11], находим, что $V Q L_{4}^{\alpha}=U(R)$, где столбцы отношения $R$, согласно рассуждению подслучая 2 , можно рассматривать как столбцы значений селекторных функций $e_{1}^{3}(x, y, z)$, $e_{2}^{3}(x, y, z), e_{3}^{3}(x, y, z)$ и некоторых трехместных функций $r(x, y, z)$ из $V Q L_{4}^{\alpha}$. Так как $f \notin U(R)$, можно выбрать $r_{1}(x, y, z), \ldots, r_{n}(x, y, z) \in V Q L_{4}^{\alpha}$ такие, что $h(x, y, z)=$ $f_{2}\left(r_{1}(x, y, z), \ldots, r_{n}(x, y, z)\right)$ не сохраняєт отношения $R$. Если $\vec{B}_{2} h=\vec{B}_{2} r$ для некоторой $r \in V Q L_{4}^{\alpha}$, то $g(x, y, z)=f_{0}\left(r(x, y, z), h(x, y, z), f_{0}(x, y, z)\right) \neq f_{0}(x, y, z)$, $\vec{B}_{2} g=\vec{B}_{2} f_{0}$. Из $g$ подстановкой переменных можно получить $g^{\prime}(x, y) \in P_{4}^{2} O_{1} \backslash O_{1}$. Согласно случаю I имеем $l_{3} \in\left[\left\{g^{\prime}\right\}\right]_{G^{*}}$. В силу работы $[11]\left[\left\{l_{3}, \gamma_{4}\right\}\right]=A_{4} \ni d$. Это означает, что ORD $[\{f\}] \leqslant 4^{3}$. Если $\vec{B}_{2} h \neq \vec{B}_{2} r$ для любой $r \in V Q L_{4}^{\alpha}$, то опять, рассуждая, как в доказательстве леммы 29 работы [11], можно получить, что $h^{\prime}(x, y, z) \in V_{4}^{2} \tilde{D}_{1} \backslash V_{4}^{2} \tilde{L}_{4}$, где $h^{\prime}(x, y, z)=\gamma_{4}(h(x, y, z), x)$, или $h^{\prime}(x, y, z)=$ $\gamma_{4}\left(h(x, y, z), f_{0}(x, y, z)\right)$. Возвращаемся к случаю II.

4. $f \in J_{3}$. Тогда согласно работе $[12]$ ORD $[\{f\}] \leqslant 3$.

5. $f \in M(k)$, где $M(3)=P_{3}^{\alpha} \backslash\left(P_{3}^{2} \vec{C}_{4} \cup J_{3}\right), M(4)=P_{4}^{\alpha} \backslash\left(P_{4}^{2} \vec{C}_{4} \cup V_{4}^{\alpha}\right)$, $M(k)=P_{k}^{\alpha} \backslash P_{k}^{2} \vec{C}_{4}$ при $k \geqslant 5$. Подстановкой переменных из $f$ можно получить $g_{1}(x, y) \in M(k)$. Если при $k=3 g_{1}(x, y) \in J_{3}$, то нетрудно видеть, что $g_{1}\left(a_{1}, a_{2}\right)=a_{3}$ при любом трехэлементном наборе $\left(a_{1}, a_{2}, a_{3}\right)$. Подстановкой переменных из $f$ можно получить $g_{2}^{\prime}(x, y, z) \in P_{3}^{\alpha} \backslash J_{3}$. Нетрудно проверить, что $g_{2}(x, y)=g_{2}^{\prime}\left(x, y, g_{1}(x, y)\right) \in P_{3}^{\alpha} \backslash J_{3}$. Если при $k=4 g_{1} \in V_{4}^{\alpha}$, т. е. $g_{1} \in V_{4}^{\alpha} \backslash V_{4}^{2} \tilde{D}_{1}$, то согласно подслучаю 3

$$
\left[\left\{g_{1}\right\}\right]_{G^{*}} \supseteq\left[\left\{\gamma_{4}\right\}\right]_{G^{*}}=A_{4}^{4} L_{4}
$$

Подстановкой переменных из $f$ можно получить $g_{2}^{\prime \prime}(x, y, z, u) \in P_{4}^{\alpha} \backslash V_{4}^{\alpha}$. Аналогично случаю $k=3$ нетрудно проверить, что в $\left[\left\{g_{2}^{\prime \prime}\right\} \cup A_{4}^{4} L_{4}\right]_{G^{*}}$ найдется 
$g_{2}(x, y) \in P_{4}^{\alpha} \backslash V_{4}^{\alpha}$. Итак, из $[\{f\}]_{G^{*}}$ всегда можно получить $g_{1}(x, y), g_{2}(x, y)$ такие, что $g_{1} \in P_{4}^{\alpha} \backslash P_{k}^{2} \vec{C}_{4}$, а $g_{2} \in P_{3}^{\alpha} \backslash J_{3}$ при $k=3$ и $g_{2} \in P_{4}^{\alpha} \backslash V_{4}^{\alpha}$ при $k=4$. Покажем, что $\left[\left\{g_{1}, g_{2}\right\}\right]_{G^{*}}$ содержит $h(x, y)$ такую, что $h(0, a)=b \in E_{k} \backslash\{0\}$ при любом $a \in E_{k} \backslash\{0\}$. Рассмотрим всевозможные варианты.

5.1. Пусть $g_{1}(x, y)$ - такая функция, что для некоторого $i \in E_{k}, g(i, j) \in$ $E_{k} \backslash\{i\}$ при любом $j \in E_{k} \backslash\{i\}$ и $g_{1}\left(i, j_{1}\right)=g_{1}\left(i, j_{2}\right)$ для некоторых $j_{1}, j_{2} \in E_{k} \backslash\{i\}$, $j_{1} \neq j_{2}$. Без ограничения общности (благодаря отношению $G^{*}$ ), можно считать, что $i=0$. Пусть

$$
\begin{aligned}
& \operatorname{Im}^{+} g(0, y)=\left\{a \in E_{k} \backslash\{0\} \mid a \in \operatorname{Im} g(0, y)\right\} \\
& \operatorname{Im}^{\oplus} g(0, y)=\left\{a \in E_{k} \backslash\{0\} \mid \operatorname{Im} g(0, a)=a\right\}
\end{aligned}
$$

Можно построить последовательность функций

$$
\tilde{g}_{2}, \ldots, \tilde{g}_{p_{1}}, \tilde{g}_{p_{1}+1}, \ldots, \tilde{g}_{p_{2}}, \tilde{g}_{p_{2}+1}, \ldots, \tilde{g}_{p_{l}}
$$

следующим образом: $\tilde{g}_{2}(x, y)=g_{1}\left(x, g_{1}(x, y)\right), \tilde{g}_{3}(x, y)=g_{1}\left(x, \tilde{g}_{2}(x, y)\right), \ldots, \tilde{g}_{p_{1}}(x, y)=$ $g_{1}\left(x, \tilde{g}_{p_{1}-1}(x, y)\right), \ldots, \tilde{g}_{p_{1}+1}(x, y)=\tilde{g}_{p_{1}}\left(x, \tilde{g}_{p_{1}}(x, y)\right), \tilde{g}_{p_{1}+2}(x, y)=\tilde{g}_{p_{1}}\left(x, \tilde{g}_{p_{1}+1}(x, y)\right), \ldots$, $\tilde{g}_{p_{2}}(x, y)=\tilde{g}_{p_{1}}\left(x, \tilde{g}_{p_{2}-1}(x, y)\right), \ldots, \tilde{g}_{p_{l}}(x, y)=\tilde{g}_{p_{l}-1}\left(x, \tilde{g}_{p_{l}-1}(x, y)\right)$, где

$$
\begin{gathered}
\left|\operatorname{Im}^{\oplus} \tilde{g}_{p_{u}}(0, y)\right| \geqslant\left|\operatorname{Im}^{\oplus} \tilde{g}_{p_{u-1}}(0, y)\right|+1 \\
\operatorname{Im}^{\oplus} \tilde{g}_{p_{u}-1}(0, y)=\operatorname{Im}^{\oplus} \tilde{g}_{p_{u-1}+1}(0, y)=\ldots=\operatorname{Im}^{\oplus} \tilde{g}_{p_{u}-1}(0, y), \quad u=2, \ldots, l,
\end{gathered}
$$

a $\operatorname{Im}^{\oplus} \tilde{g}_{p_{l}}(0, y)=\operatorname{Im}^{+} \tilde{g}_{p_{l}}(0, y)$. Легко видеть, что $\tilde{g}_{p_{l}}\left(0, a_{1}\right)=\ldots=\tilde{g}_{p_{l}}\left(0, a_{i_{1}}\right)=$ $a_{1}, \ldots, \tilde{g}_{p_{l}}\left(0, a_{i_{m-1}+1}\right)=\ldots=\tilde{g}_{p_{l}}\left(0, a_{i_{m}}\right)=a_{i_{m-1}}+1$, где $\left\{a_{1}, \ldots, a_{i_{m}}\right\}=E_{k} \backslash\{0\}$, $\mathrm{Im}^{+} \tilde{g}_{p_{l}}(0, y)=\left\{a_{1}, a_{i_{1}+1}, \ldots a_{i_{m-1}+1}\right\}$. Без ограничения общности можно предположить, что $i_{1} \geqslant 2$. Поскольку $\left|\operatorname{Im} \tilde{g}_{p_{l}}(0, y)\right| \leqslant k-2$, аналогично доказательству лемм 2 и 3 можно получить $\tilde{g}_{p_{l}, 1}(x, y)$ из $\left[\left\{\tilde{g}_{p_{l}, 1}\right\}\right]_{G^{*}}$ такую, что $\tilde{g}_{p_{l}, 1}(0, y)=a_{1}$ при $y \in E_{k} \backslash\{0\}$. Тогда $\tilde{g}_{p_{l}, 1}$ является искомой $h(x, y)$.

5.2. Пусть $g_{1}(x, y)$ - такая функция, что $g_{1}\left(i, j_{1}\right)=i, g_{1}\left(i, j_{2}\right) \neq i$ для некоторых $i, j_{1}, j_{2} \in E_{k},\left|\left\{i, j_{1}, j_{2}\right\}\right|=3$. Без ограничения общности можно считать, что $i=0$.

Если $g_{1}\left(0, j_{2}\right)=j_{3} \in E_{k} \backslash\left\{j_{2}, 0\right\}$, то в $G$ найдется $\pi=(0)\left(j_{2} j_{3}\right)(\ldots) \ldots(\ldots)$. Пусть $g_{1}^{1}(x, y)=g_{1}\left(x, g_{1}^{\pi}(x, y)\right)$. Нетрудно видеть, что $g_{1}^{1}\left(0, j_{3}\right)=j_{3}$. Аналогично ситуации 5.1 из $\left[\left\{g_{1}^{1}\right\}\right]_{G^{*}}$ можно получить, без ограничения общности, такую $\tilde{g}_{1}$, чTO

$$
\begin{aligned}
\tilde{g}_{1}(0,1) & =\ldots=\tilde{g}_{1}\left(0, i_{1}\right)=1, \\
\tilde{g}_{1}\left(0, i_{1}+1\right)= & \ldots=\tilde{g}_{1}\left(0, i_{1}+i_{2}\right)=i_{1}+1, \\
& \ldots \\
\tilde{g}_{1}\left(0, i_{1}+\ldots+i_{s-1}+1\right)= & \ldots=\tilde{g}_{1}\left(0, i_{1}+\ldots+i_{s}\right)=i_{1}+\ldots+i_{s-1}+1, \\
\tilde{g}_{1}\left(0, i_{1}+\ldots+i_{s}+1\right)= & i_{1}+\ldots+i_{s}+1, \\
\tilde{g}_{1}\left(0, i_{1}+\ldots+i_{s}+2\right)= & i_{1}+\ldots+i_{s}+2, \\
& \ldots \\
\tilde{g}_{1}(0, q)= & q, \tilde{g}_{1}(0, q+1)=0, \ldots, \tilde{g}_{1}(0, k-1)=0 .
\end{aligned}
$$


Для любой $g(x, y)$ пусть

$$
\begin{aligned}
\Lambda_{g}^{i} & =(g(i, 0), \ldots, g(i, i-1), g(i, i+1), \ldots, g(i, k-1)), \\
I_{g}^{i}(a) & =\left|\left\{b \in E_{k} \backslash\{i\} \mid g(i, b)=a\right\}\right|, \\
r_{+}^{i}(g) & =\sum_{a \in \operatorname{Im}}\left(I_{g(i, y) \backslash\{i\}}\left(I_{g}^{i}(a)-1\right) .\right.
\end{aligned}
$$

(a) Пусть $r_{+}^{0}\left(\tilde{g}_{1}\right)=0, I_{\tilde{g}_{1}}^{0}(0)=1$, т. е. $\Lambda_{\tilde{g}_{1}}^{0}=(1,2, \ldots, k-2,0)$. Из $\left[\left\{\tilde{g}_{1}\right\}\right]_{G^{*}}$ можно выбрать $\tilde{g}_{1,1}(x, y)$ такую, что $\tilde{g}_{1,1}(0, k-1)=1$. Пусть $\tilde{g}_{1,2}(x, y)=\tilde{g}_{1,1}\left(\tilde{g}_{1}(x, y), y\right)$. Тогда $\Lambda_{\tilde{g}_{1,2}}^{0}=(1,2, \ldots, k-2,1)$. Возвращаемся к подслучаю $\mathrm{I}$.

(b) Пусть $r_{+}^{0}\left(\tilde{g}_{1}\right)=0, I_{\tilde{g}_{1}}^{0}(0)>1$, т. е. $\Lambda_{\tilde{g}_{1}}^{0}=(1, \ldots, q, 0, \ldots, 0), q<k-2$. В $G$ всегда найдется $\pi_{1}$ такая, что $\pi_{1}(0)=0,\left\{\pi_{1}(2), \ldots, \pi_{1}(q+1)\right\}=\{1, \ldots, q\}$. Пусть $\tilde{g}_{1,3}=\left\langle\pi_{1}\right\rangle\left(\tilde{g}_{1}\right), \tilde{g}_{1,4}=\tilde{g}_{1}\left(\tilde{g}_{1,3}(x, y), y\right)$. Тогда $\Lambda_{\tilde{g}_{1,4}}^{0}=(1,2, \ldots, q+1,0, \ldots, 0)$. Продолжая эту процедуру, возвращаемся к подслучаю (a).

(c) Пусть $r_{+}^{0}\left(\tilde{g}_{1}\right) \geqslant 1, I_{\bar{g}_{1}}^{0}(0) \geqslant 1$. Аналогично доказательствам лемм 2 и 3 в

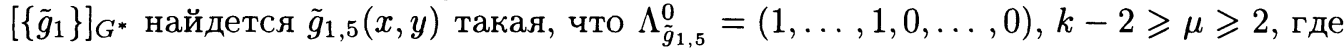
$\mu$ - число единиц в векторе.

Предположим, что $\left[\left\{\tilde{g}_{1,5}\right\}\right]_{G^{*}}$ содержит $\tilde{g}_{1,5}, 0(x, y)$ такую, что $\tilde{g}_{1,5}^{0}(0,1)=$ $\tilde{g}_{1,5}^{0}(1,0)=1 . \quad \mathrm{B}\left[\left\{\tilde{g}_{1,5}\right\}\right]_{G^{*}}$ найдется $\tilde{g}_{1,5}^{1}(x, y)$ такая, что $\tilde{g}_{1,5}^{1}(0, \mu+1)=1$ и $\tilde{g}_{1,5}^{1,5}(0, a) \in\{0,1\}$ при любом $a \in E_{k} \backslash\{0\}$. Для $\tilde{g}_{1,5}^{2}(x, y)=\tilde{g}_{1,5}^{0}\left(\tilde{g}_{1,5}(x, y), \tilde{g}_{1,5}^{1}(x, y)\right)$

$$
\Lambda_{\tilde{g}_{1,5}^{2}}^{0}=\left(1, \ldots, 1, e_{\mu+2}, \ldots, e_{k-1}\right),
$$

где первые $\mu+1$ элемента равны единице и $e_{\mu+2}, \ldots, e_{k-1} \in\{0,1\}$. Продолжая эту процедуру, можно получить $\tilde{g}_{1,6}$ из $\left[\left\{\tilde{g}_{1,5}\right\}\right]_{G^{*}}$ такую, что $\Lambda_{\tilde{g}_{1,6}}^{0}=(1, \ldots, 1)$.

Если $\tilde{g}_{1,5}\left(\rho_{1}, 0\right)=\rho_{2} \neq \rho_{1}$ для некоторого $\rho_{1} \in\{\mu+1, \ldots, k-1\}$, то пусть $\tilde{g}_{1,5}^{4}(x, y)=\tilde{g}_{1,5}^{3}\left(x, \tilde{g}_{1,5}(x, y)\right)$, где $\tilde{g}_{1,5}^{3} \in\left[\left\{\tilde{g}_{1,5}(x, y)\right\}\right]_{G^{*}}$ и $\tilde{g}_{1,5}^{3}\left(\rho_{1}, \rho_{2}\right)=0$. Тогда $\tilde{g}_{1,5}^{4}\left(0, \rho_{1}\right)=\tilde{g}_{1,5}^{4}\left(\rho_{1}, 0\right)=0 . \quad \mathrm{B}\left[\left\{\tilde{g}_{1,5}^{4}\right\}\right]_{G^{*}}$ всегда найдется $\tilde{g}_{1,5}^{5}(x, y)$ такая, что $\tilde{g}_{1,5}^{5}(0,1)=\tilde{g}_{1,5}^{5}(1,0)=1$. Возвращаемся к рассмотренной ситуации.

Предположим, что $\tilde{g}_{1,5}(1,0)=0, \tilde{g}_{1,5}(\rho, 0)=0$ для любого $\rho \in\{\mu+1, \ldots, k-1\}$.

Пусть $\mu \geqslant[k / 2]$. В $G$ найдется $\pi_{2}$ такая, что $\pi_{2}(0)=0, \pi_{2}(k-q)=q$, $q=1, \ldots,[k / 2] . \quad$ Пусть $\tilde{g}_{1,5}^{6}=\tilde{g}_{1,5}^{\pi_{2}}, \tilde{g}_{1,5}^{7}(x, y)=\tilde{g}_{1,5}\left(\tilde{g}_{1,5}^{6}(x, y), \tilde{g}_{1,5}^{5}(x, y)\right)$. Если $\tilde{g}_{1,5}(k-1,1) \neq 0$, то легко проверить, что $\tilde{g}_{1,5}^{7}(0, y) \neq 0$ при любом $y \in E_{k} \backslash\{0\}$, кроме того, $\tilde{g}_{1,5}^{7}(0, k-2)=\tilde{g}_{1,5}^{7}(0, k-1)=k-1$. Возвращаемся к подслучаю 5.1 . Если $\tilde{g}_{1,5}(k-1,1)=0$, то положим $\tilde{g}_{1,5}^{8}(x, y)=\tilde{g}_{1,5}\left(\tilde{g}_{1,5}^{7}(x, y), \tilde{g}_{1,5}(x, y)\right)$. Легко проверить, что $\tilde{g}_{1,5}^{8}(0, a) \in\{1, k-1\}$ для любого $a \in E_{k} \backslash\{0\}$. Возвращаемся к подслучаю 5.1 .

Пусть $2 \leqslant \mu<[k / 2]$. В $\left[\left\{\tilde{g}_{1,5}\right\}\right]_{G^{*}}$ найдется $\tilde{g}_{1,5}^{9}(x, y)$ такая, что $\tilde{g}_{1,5}^{9}(0,1)=$ $\tilde{g}_{1,5}^{9}(0, k-1)=1$. Пусть $\tilde{g}_{1,5}^{10}(x, y)=\tilde{g}_{1,5}^{9}\left(x, \tilde{g}_{1,5}^{7}(x, y)\right)$. Легко проверить, что $\tilde{g}_{1,5}^{10}(0, a)=1$ при $a \in\{1, \ldots, \mu, k-1, k-2, \ldots, k-\mu\}$. Если $2 \mu \geqslant[k / 2]$, то с функцией $\tilde{g}_{1,5}^{10}$ возвращаемся к предыдущей ситуации. Если $2 \mu<[k / 2]$, то аналогичным рассуждением можно получить $\tilde{g}_{1,5}^{11}$ из $\left[\left\{\tilde{g}_{1,5}\right\}\right]_{G^{*}}$ такую, что $\tilde{g}_{1,5}^{11}(0, y)$ принимает $4 \mu$ раз значение 1 и $k-1-4 \mu$ раз значение 0, кроме того $\tilde{g}_{1,5}^{11}(0,1)=1$. Продолжая такую процедуру, всегда возвращаемся к рассмотренной ситуации.

5.3. Пусть для некоторых $i, j \in E_{k}, i \neq j$, имеют место соотношения $\Lambda_{g_{1}}^{i}=$ $(i, \ldots, i), \mid\left\{j, g_{1}\left(j, a_{1}\right), g_{1}\left(j, a_{2}\right) \mid=3\right.$ при любых $a_{1}, a_{2} \in E_{k} \backslash\{j\}, a_{1} \neq a_{2}$. Если $g_{1}(j, b)=b$ для некоторого $b \in E_{k} \backslash\{j\}$, то при $\vec{g}_{1}(x, y)=g_{1}(y, x)$

$$
\vec{g}_{1}(b, j)=b, \quad \vec{g}_{1}(b, i)=i, \quad \vec{g}_{1}(x, y) \in P_{k}^{\alpha} \backslash P_{k}^{2} \vec{C}_{4}
$$


Возвращаемся к ситуации 5.2. В противном случае пусть

$$
g_{1,1}(x, y)=g_{1}\left(x, g_{1}(x, y)\right), \ldots, g_{1, q+1}(x, y)=g_{1}\left(x, q_{1, q}(x, y)\right), \ldots
$$

Тогда $g_{1, s}(i, a)=i, g_{1, s}(j, a)=a$ для некоторых $s, a, s \leqslant k, a \in E_{k} \backslash\{i, j\} . \mathrm{C}$ функциями $\vec{g}_{1, k}(x, y)=g_{1, k} \cdot(y, x)$ и $g_{1}(x, y)$ возвращаемся к ситуации 5.2 .

5.4. Пусть $g_{1}(i, a) \neq i, g_{1}\left(i, a_{1}\right) \neq g_{1}\left(i, a_{2}\right)$ для любых $i, a, a_{1}, a_{2}$, где $a_{1} \neq a_{2}$, $i \notin\left\{a, a_{1}, a_{2}\right\}$. Так как $g_{1} \in P_{k}^{\alpha} \backslash P_{k}^{2} \vec{C}_{4}$, имеем $g_{1}(j, b)=c \in E_{k} \backslash\{j, b\}$ для некоторых $j, b \in E_{k}$.

(a) Если $g_{1}\left(j_{1}, b\right)=g_{1}\left(j_{2}, b\right)$ или $g_{1}\left(j_{3}, b\right)=b$ для некоторых $j_{1}, j_{2}, j_{3} \in E_{k}$, $j_{1} \neq j_{2}$, то с функцией $\vec{g}_{1}(x, y)=g_{1}(y, x)$ возвращаемся либо к ситуации 5.1 , либо к ситуации 5.2 .

В противном случае, при $k=3$

$$
\Lambda_{g_{1}}^{0}=(2,1), \quad \Lambda_{g_{1}}^{1}=(2,0), \quad \Lambda_{g_{1}}^{2}=(1,0),
$$

а при $k=4$ либо

$$
\Lambda_{g_{1}}^{0}=(2,3,1), \quad \Lambda_{g_{1}}^{1}=(3,0,2), \quad \Lambda_{g_{1}}^{2}=(1,3,0), \quad \Lambda_{g_{1}}^{3}=(2,1,0)
$$

либо

$$
\Lambda_{g_{1}}^{0}=(3,1,2), \quad \Lambda_{g_{1}}^{1}=(2,3,0), \quad \Lambda_{g_{1}}^{2}=(3,0,1), \quad \Lambda_{g_{1}}^{3}=(1,2,0) .
$$

Аналогично рассуждению, имеющемуся в начале этого подслучая 5 , из $g_{1}$, $g_{2} G^{*}$-замыканием можно получить $g_{1}^{*}(x, y)$ такую, что $g_{1}^{*} \in P_{k}^{\alpha} \backslash P_{k}^{2} \vec{C}_{4}$ и относительно $g_{1}^{*}$ не верна ни одна из систем равенств $(1),(2),(3)$, т. е. возвращаемся к рассмотренным ситуациям.

Пусть $k \geqslant 5$. Покажем, что в $\left[\left\{g_{1}\right\}\right]_{G^{*}}$ либо найдется $g_{1}^{*}(x, y)$ такая, что $g_{1}^{*}\left(b, j_{4}\right)=j_{4}, g_{1}^{*}(b, j)=c \in E_{k} \backslash\{b, j\}$ для некоторых $j_{4}, j \in E_{k} \backslash\{b\}$ и $\mid\left\{g_{1}^{*}\left(b, j_{1}\right)\right.$, $\left.g_{1}^{*}\left(b, j_{2}\right), b\right\} \mid=3$ для любых $j_{1}, j_{2} \in E_{k} \backslash\{b\}, j_{1} \neq j_{2}$, либо найдется $g_{1}^{* *}(x, y)$, с которой возвращаемся к рассмотренным ситуациям.

$\mathrm{B}\left[\left\{\vec{g}_{1}(x, y)\right\}\right]_{G^{*}}$ всегда найдется $\vec{g}_{1,1}(b, y)$ такая, что РНЩ $\vec{g}_{1,1}(x, y)$ содержит только циклы длины 1 и $p_{1}$, где $p_{1}$ - простое число, и $\vec{g}_{1,1}(b, y)$ нетривиальна. Если РНЦ $g_{1,1}(b, y)$ содержит хотя бы один цикл длины 1 , то $\vec{g}_{1,1}$ является искомой $g_{1}^{*}$. Пусть РНЦ $\vec{g}_{1,1}(b, y)$ содержит только циклы длины $p_{1}$. Тогда $k-1=n_{1} p_{1}$.

Пусть $G_{b}=\{\varphi(x) \in G \mid \varphi(b)=b\}$. Если $n_{1}=1, p_{1} \geqslant 5$ или $k-1 \geqslant 10$, то, аналогично доказательству леммы 1 , в силу 2 -транзитивности $\left[\left\{\vec{g}_{1,1}(b, y)\right\}\right]_{G_{b}^{*}}$ в $\left[\left\{\vec{g}_{1,1}(x, y)\right\}\right]_{G_{b}^{*}}$ найдется искомая функция $g_{1}^{*}$. Предположим, что $n_{1} \geqslant 2$. Если $\left(k-1, p_{1}\right) \in\{(8,2),(9,3)\}$, то из доказательства леммы 1 нетрудно получить $g_{1}^{*}$ из $\left[\left\{\vec{g}_{1,1}(x, y)\right\}\right]_{G_{b}^{*}}$. Остаются ситуации, когда $\left(k-1, p_{1}\right) \in\{(4,2),(6,3),(6,2)\}$.

Пусть $\left(k-1, p_{1}\right)=(4,2)$. Без ограничения общности предположим, что $b=0$ и $\Lambda_{g_{1,1}}^{0}=(2,1,4,3)$. Если $\vec{g}_{1,1}(j, y)=y$ для некоторого $j \in E_{5}$, то с функцией $\overrightarrow{\vec{g}}_{1,1}(x, y)=\vec{g}_{1,1}(y, x)$ возвращаемся к ситуации 5.2. Если подгруппа $\left[\left\{\vec{g}_{1,1}(j, y)\right\}\right]_{G_{j}^{*}}$ нечетверна и неединична для некоторого $j$, то легко получить $g_{1}^{*}$ из $\left[\left\{\vec{g}_{1,1}\right\}\right]_{G_{j}^{*}}$. Предположим, что $\vec{g}_{1,1}(i, y)$ - нетривиальная перестановка четверной группы для любого $i \in E_{k}$. Если $\vec{g}_{1,1}(4,3) \in\{1,2,3,4\}$, то в силу того, что $\overrightarrow{\vec{g}}_{1,1}(3,0)=4$, $\overrightarrow{\vec{g}}_{1,1}(3,4) \in\{1,2,3,4\}$, приходим либо к одной из ситуаций $5.1,5.2$, либо к тому, что $\left[\left\{\overrightarrow{\vec{g}}_{1,1}(3, y)\right\}\right]_{G_{3}^{*}}$ нечетверна и неединична, т. е. к рассмотренной ситуации. 
Пусть $\vec{g}_{1,1}(4,3)=0$. Тогда либо мы имеем неединичную, нечетверную подгруппу $\left[\left\{\vec{g}_{1,1}(4, y)\right\}\right]_{G_{4}^{*}}$, либо $\Lambda_{\vec{g}_{1,1}}^{4}=(3,2,1,0)$ (тогда $\overrightarrow{\vec{g}}_{1,1}(1,0)=\overrightarrow{\vec{g}}_{1,1}(1,4)=2$ ). Возвращаемся к рассмотренным ситуациям.

Пусть $\left(k-1, p_{1}\right)=(6,3)$. Без ограничения общности предположим, что $\Lambda_{\vec{g}_{1,1}}^{0}=$ $(2,3,1,5,6,4)$. В $G_{0}$ найдется

$$
\theta_{1}=\left(\begin{array}{ccccccc}
0 & 1 & 4 & 3 & * & * & * \\
0 & 1 & 3 & 2 & * & * & *
\end{array}\right)
$$

такая, что для $\vec{g}_{1,2}(x, y)=\vec{g}_{1,1}\left(x, \vec{g}_{1,1}^{\theta_{1}}(x, y)\right)$ справедливы равенства $\vec{g}_{1,2}(0,1)=1$, $\vec{g}_{1,2}(0,4)=2$, т. е. $\vec{g}_{1,2}$ является искомой $g_{1}^{*}$.

Пусть $\left(k-1, p_{1}\right)=(6,2)$. Без ограничения общности предположим, что $\Lambda_{\vec{g}_{1,2}}^{0}=$ $(2,1,4,3,6,5)$. В $G_{0}$ найдется

$$
\theta_{2}=\left(\begin{array}{ccccccc}
0 & 1 & 4 & 6 & * & * & * \\
0 & 4 & 3 & 1 & * & * & *
\end{array}\right)
$$

Тогда $\vec{g}_{1,1}^{\theta_{2}}(0,1)=4, \vec{g}_{1,1}^{\theta_{2}}(0,4)=1$ и $\theta_{2}^{-1}(2) \in\{5,3,2\}$. Если $\theta_{2}(5)=2$, то для $\vec{g}_{1,3}(x, y)=\vec{g}_{1,1}\left(x, \vec{g}_{1,1}^{\theta_{2}}(x, y)\right)$ справедливы разенства $\vec{g}_{1,3}(0,1)=3, \vec{g}_{1,3}(0,6)=6$, т. е. $\vec{g}_{1,3}$ является искомой $g_{1}^{*}$.

Пусть $\theta_{2}(2)=2$ или $\theta_{2}(2)=3$. Если РНЦ $\vec{g}_{1,1}^{\theta_{2}}$, содержит цикл длины, не равной 2 , то возвращаемся к рассмотренным ситуациям. Если РНЦ $\vec{g}_{1,1}^{\theta_{2}}$ содержит только цикл длины 2 , то легко видеть, что $\Lambda_{\vec{g}_{1,1}^{\theta_{2}}}^{0}=(4,5,6,1,2,3)$ или $\Lambda_{\vec{g}_{1,1}^{\theta_{2}}}^{0}=(4,6,5,1,3,2)$. Пусть $\vec{g}_{1,3}(x, y)=\vec{g}_{1,1}\left(x, \vec{g}_{1,1}^{\theta_{2}}(x, y)\right)$. Легко видеть, что РНЦ $\vec{g}_{1,3}(0, y)$ всегда содержит тройной цикл. Возвращаемся к рассмотренным ситуациям.

Пусть $\vec{g}_{1}^{*}(x, y)=g_{1}^{*}(x, y)$. Если $\Lambda_{\vec{g}_{1}^{*}}^{j_{4}}=\left(j_{4}, \ldots, j_{4}\right)$, то в силу справедливости равенства $\vec{g}_{1}^{*}(j, b)=c$, возвращаемся к одной из ситуаций $5.2,5.3$. В противном случае возвращаемся к ситуации 5.2 .

Дальше докажем, что $C_{k, 1}^{2} \in[\{h\}]_{G^{*}}$. Очевидно, что $[\{h\}]_{G^{*}}$ содержит $h_{1}(x, y)$ такую, что $\Lambda_{h_{1}}^{0}=(1, \ldots, 1)$.

(А) Пусть $h_{1}(x, y)$ такая, что $\Lambda_{h_{1}}^{0}=(1, \ldots, 1), \Lambda_{h_{1}}^{s}=(\varepsilon, \ldots, s), s=1, \ldots, k-1$. Предположим, что $\left[\left\{h_{1}\right\}\right]_{G^{*}}$ содержит $h_{1}^{l}(x, y)$ такую, что $\Lambda_{h_{1}^{l}}^{s}=(1, \ldots, 1), s=$ $0, \ldots, l, 0 \leqslant l<k-1, \Lambda_{h_{1}^{l}}^{r}=(r, \ldots, r), r=l+1, \ldots, k-1$. Нетрудно видеть, что $\left[\left\{h_{1}^{l}\right\}\right]_{G^{*}}$ содержит $h_{1}^{l, 1}(x, y)$ такую, что $\Lambda_{h_{1}^{l, 1}}=(0, \ldots, 0), \Lambda_{h_{1}^{l, 1}}^{s}=(1, \ldots, 1)$, $s=1, \ldots, l+1, \Lambda_{h_{1}^{l, 1}}^{r}=(r, \ldots, r), r=l+2, \ldots, k-1$. Пусть $h_{1}^{l+1}(x, y)=$ $h_{1}^{l}\left(h_{1}^{l, 1}(x, y), h_{1}^{l}(x, y)\right)$. Тогда

$$
\Lambda_{h_{1}^{l+1}}^{s}=(1, \ldots, 1), \quad s=0, \ldots, l+1, \quad \Lambda_{h_{1}^{l+1}}^{r}=(r, \ldots, r), \quad r=l+2, \ldots, k-1 .
$$

Таким индуктивным построением получим, что $C_{k, 1}^{2} \in\left[\left\{h_{1}\right\}\right]_{G^{*}}$.

(В) Пусть $h_{1}\left(i, j_{1}\right)=i \neq h_{1}\left(i, j_{2}\right)$ для некоторых $i, j_{1}, j_{2} \in E_{k},\left|\left\{i, j_{1}, j_{2}\right\}\right|=3$. Для определенности пусть $i=1$. 
Если $h_{1}(1,0)=t_{1} \neq i$, то $1 \neq j_{1} \neq 0$. Если $h_{1}\left(j_{1}, 0\right)=j_{1}$, то пусть

$$
\begin{array}{rlrl}
\pi_{3} & =\left(10 j_{1}\right)(\ldots) \ldots(\ldots) \in G, & & h_{1,1}(x, y)=h_{1}^{\pi_{3}}\left(x, h_{1}(x, y)\right), \\
\pi_{4}=(01)(\ldots) \ldots(\ldots) \in G, & & h_{1,2}(x, y)=h_{1}^{\pi_{4}}\left(x, h_{1}(x, y)\right), \\
h_{1,3}(x, y) & =h_{1}\left(h_{1,1}(x, y), h_{1,2}(x, y)\right), & & h_{1,4}(x, y)=h_{1}\left(x, h_{1,3}(x, y)\right) .
\end{array}
$$

Легко проверить, что $\Lambda_{h_{1,4}}^{0}=(1, \ldots, 1), \Lambda_{h_{1,4}}^{1}=(1, \ldots, 1)$. Если $h_{1}\left(j_{1}, 0\right)=j_{3} \neq j_{1}$, то пусть $h_{1,5}(x, y)=h_{1}\left(x, h_{1,1}(x, y)\right)$. Легко проверить, что $\Lambda_{h_{1,5}}^{0}=(1, \ldots, 1)$, $\Lambda_{h_{1,5}}^{1}=(1, \ldots, 1)$. Если $h_{1}(1,0)=1$, то пусть $h_{1,6}(x, y)=h_{1}\left(h_{1,2}(x, y), h_{1,1}(x, y)\right)$. Легко проверить, что $\Lambda_{h_{1,6}}^{0}=(1, \ldots, 1), \Lambda_{h_{1,6}}^{1}=(1, \ldots, 1)$. Итак, в этом случае $\left[\left\{h_{1}\right\}\right]_{G^{*}}$ всегда содержит $h_{2}(x, y)$ такую, что $\Lambda_{h_{2}}^{0}=(1, \ldots, 1), \Lambda_{h_{2}}^{1}=(1, \ldots, 1)$. Нетру,дно видеть, что $\left[\left\{h_{2}\right\}\right]_{G^{*}}$ содержит $h_{2,1}(x, y), h_{2,2}(x, y)$ такие, что $\Lambda_{h_{2,1}}^{0}=$ $(0, \ldots, 0), \Lambda_{h_{2,1}}^{2}=(0, \ldots, 0), \Lambda_{h_{2,2}}^{1}=(1, \ldots, 1), \Lambda_{h_{2,2}}^{2}=(1, \ldots, 1)$. Пусть $h_{2,3}(x, y)=$ $h_{2,2}\left(x, h_{2,1}(x, y)\right) . \quad$ Тогда $\Lambda_{h_{2,3}}^{0}=(0, \ldots, 0), \Lambda_{h_{2,3}}^{1}=(1, \ldots, 1), \Lambda_{h_{2,3}}^{2}=(1, \ldots, 1)$. Предпюложим, что $\left[\left\{h_{2}\right\}\right]_{G^{*}}$ содержит $h_{1}(x, y)$ такую, что $\Lambda_{h_{1}}^{s, 3}=(s, \ldots, s)$, $s=0, \ldots, l-1, \Lambda_{h_{l}}^{l}=(l-1, \ldots, l-1), 2 \leqslant l \leqslant k-2$. Используя предложение 1 , легко проверить, что $\left[\left\{h_{l}\right\}\right]_{G^{*}}$ содержит $h_{l, 1}(x, y), h_{l, 2}(x, y)$ такие, что $\Lambda_{h_{l, 1}}^{s}=(s, \ldots, s)$, $s=0, \ldots, l-1, \Lambda_{h_{l, 1}}^{l+1}=(l-1, \ldots, l-1)$, a $\Lambda_{h_{l, 2}}^{l}=(l, \ldots, l), \Lambda_{h_{l, 2}}^{l+1}=(l, \ldots, l)$. Пусть $h_{l+1}(x, y)=h_{l, 2}\left(x, h_{l, 1}(x, y)\right)$. Легко видеть, что $\Lambda_{h_{l+1}}^{s}=(s, \ldots, s), s=0, \ldots, l$, $\Lambda_{h_{l+1}}^{l+1}==(l, \ldots, l)$. Тем самым доказано, что $\left[\left\{h_{1}\right\}\right]_{G^{*}} \ni h_{k-1}(x, y)$, где $\Lambda_{h_{k-1}}^{s}=$ $(s, \ldots, s), s=0, \ldots, k-2, \Lambda_{h_{k-1}}^{k-1}=(k-2, \ldots, k-2)$. Пусть

$$
\pi_{5}=\left(\begin{array}{ccccc}
0 & 1 & * & \ldots & * \\
k-1 & k-2 & * & \ldots & *
\end{array}\right) \in G, \quad h_{k}=h_{k-1}^{\pi_{5}} .
$$

Тогда $\Lambda_{h_{k}}^{0}=(1, \ldots, 1), \Lambda_{h_{k}}^{s}=(s, \ldots, s), s=1, \ldots, k-1$. Возвращаемся к случаю $\mathrm{A}$.

(C) Пусть $h_{1}(i, j) \neq i$ для любых $i, j \in E_{k}, i \neq j$. Очевидно, что $\left[\left\{h_{1}\right\}\right]_{G^{*}}$ содержит $h_{s}^{*}(x, y), s=0, \ldots, k-1$, такие, что $\Lambda_{h_{s}^{*}}^{s}=\left(t_{s}, \ldots, t_{s}\right), t_{s} \neq s, h_{s}^{*}(i, j) \neq i$ для любых $i, j \in E_{k}, i \neq j$. Пусть

$$
\begin{aligned}
h_{1,1}^{*}(x, y) & =h_{1}^{*}\left(x, h_{1}(x, y)\right), \\
h_{1,2}^{*}(x, y) & =h_{2}^{*}\left(x, h_{1,1}^{*}(x, y)\right), \\
& \ldots \\
h_{1, k-1}^{*}(x, y) & =h_{k-1}^{*}\left(x, h_{1, k-2}^{*}(x, y)\right) .
\end{aligned}
$$

Если $h_{1, s}^{*}\left(i_{1}, y\right)=i_{1}, h_{1, s}\left(i_{2}, y\right)=c \neq i_{2}$ для некоторых $i_{1}, i_{2}, c, s \in E_{k}, i_{1} \neq i_{2}$, $s \neq 0$, или $h_{1, s}^{*}\left(i, j_{1}\right)=i \neq h_{1, s}^{*}\left(i, j_{2}\right)$ для некоторых $i, j_{1}, j_{2}, s \in E_{k},\left|\left\{i, j_{1}, j_{2}\right\}\right|=3$, $s \neq 0$, то возвращаемся к ситуациям, рассмотренным в случае В. В противном случае $h_{1, k-1}^{*}(x, y)$ должна быть такой функцией, что $\Lambda_{h_{1, k-1}^{*}}^{s}=\left(v_{s}, \ldots, v_{s}\right), v_{s} \in$ $E_{k}, s=0, \ldots, k-1$ и $v_{k-1} \neq k-1$. Если $v_{a}=v_{b}$ для некоторых $a, b \in E_{k}$, $a \neq b$, то рассуждая аналогично схеме, изложенной в ситуации 5.1 , получим, что $C_{k, v_{a}}^{2} \in\left[\left\{h_{1, k-1}^{*}\right\}\right]_{G^{*}}$. В противном случае пусть $S_{x, y}^{\alpha}$ есть множество всех таких двуместных функций $g(x, y)$ из $P_{k}^{\alpha}$, что $\Lambda_{g}^{s}=\left(v_{s}, \ldots, v_{s}\right), v_{s} \in E_{k}, s=0, \ldots, k-1$, и $v_{a} \neq v_{b}$ для любых $a, b \in E_{k}, a \neq b$. Пусть

$$
A_{s}=\{(s, 0), \ldots,(s, s-1),(s, s+1), \ldots,(s, k-1)\}, \quad s=0, \ldots, k-1 .
$$


Пусть $\hat{A}_{k}=\left\{A_{0}, \ldots, A_{k-1}\right\}$. Построим некоторое отображение $F$ из $S_{x, y}^{\alpha}$ на $S\left(\hat{A}_{k}\right)$ следующим образом: для каждой $g \in S_{x, y}^{\alpha}$ перестановка $\hat{g}=F(g)$ такова, что $\hat{g}\left(A_{i}\right)=A_{j}$ имеет место для любых $i, j \in E_{k}$ тогда и только тогда, когда $g(x, y)=j$ при $(x, y) \in A_{i}$. Используем обозначение $\hat{g}(X)$, где $X$ - аргумент функции $\hat{g}$. Для каждой перестановки

$$
\pi=\left(\begin{array}{ccc}
0, & \ldots, & k-1 \\
i_{0}, & \ldots, & i_{k-1}
\end{array}\right)
$$

пусть

$$
\hat{\pi}=\left(\begin{array}{ccc}
A_{0}, & \ldots, & A_{k-1} \\
A_{i_{0}}, & \cdots, & A_{i_{k-1}}
\end{array}\right) .
$$

Нетрудно проверить, что

$(\alpha)$ для любых $\pi \in S\left(E_{k}\right), g \in S_{x, y}^{\alpha}$ верно, что $F\left(g^{\pi}\right)=\hat{g}^{\pi}$,

( $\beta$ ) для любых $g^{1}, g^{2}$ из $S_{x, y}^{\alpha}$ верно, что $F\left(g^{1}\left(g^{2}(x, y), y\right)\right)=\hat{g}^{1}\left(\hat{g}^{2}(X)\right)$,

$(\gamma)$ для каждой $\hat{g} \in S\left(\hat{A}_{k}\right)$ существует единственная $g \in S_{x, y}^{\alpha}$ такая, что $F(g)=\hat{g}$ и, если $\hat{g}$ порождается элементами $\hat{g}_{1}, \ldots, \hat{g}_{m}$, то существует $g \in\left[\left\{g_{1}, \ldots g_{m}\right\}\right]_{G^{*}}$ такая, что $F(g)=\hat{g}$.

Для любых $\hat{g}_{1}, \ldots, \hat{g}_{m} \in S\left(\hat{A}_{k}\right)$ пусть $\left[\left\{\hat{g}_{1}, \ldots, \hat{g}_{m}\right\}\right]_{G^{*}}$ - минимальная подгруппа всех инвариантных подгрупп группы $S\left(\hat{A}_{k}\right)$, содержащих $\left\{\hat{g}_{1}, \ldots, \hat{g}_{m}\right\}$. При $k \geqslant 3, k \neq 4$, в силу леммы $1,\left[\left\{\hat{h}_{1, k-1}^{*}\right\}\right]_{G^{*}} 2$-транзитивна. При $k=4$ пусть $h_{1,4}^{*}(x, y)=g_{2}\left(x, h_{1,3}^{*}(x, y)\right)$. Нетрудно проверить, что либо $A\left(\hat{A}_{4}\right) \subseteq\left[\left\{\hat{h}_{1,3}^{*}\right\}\right]_{G^{*}}$, либо $A\left(\hat{A}_{4}\right) \subseteq\left[\left\{\hat{h}_{1,4}^{*}\right\}\right]_{G^{*}}$. Отсюда следует, что при $k \geqslant 3$ в $\left[\left\{\hat{h}_{1, k-1}^{*}, g_{2}\right\}\right]_{G^{*}}$ всегда найдутся $t_{1}(x, y), t_{2}(x, y) \in S_{x, y}^{\alpha}$ такие, что $t_{1}=t_{2}=1$ на $A_{0}, t_{1}=0, t_{2}=2$ на $A_{1}$. Пусть $t_{3}(x, y)=t_{1}\left(t_{1}(x, y), t_{2}(x, y)\right)$. Тогда $t_{3}=1$ на $A_{0} \cup A_{1}$. Возвращаемся к ситуации, рассмотренной в случае В.

Итак, доказано, что $C_{k, 1}^{2} \in\left[\left\{g_{1}, g_{2}\right\}\right]_{G^{*}}$. Легко видеть, что $C_{k, \gamma}^{2} \in\left[\left\{C_{k, 1}^{2}\right\}\right]_{G^{*}}$ при любом $\gamma \in E_{k}$. Пусть

$$
M Z_{3}\left(a_{1}, a_{2}, \ldots, a_{s}\right)=\left\{m(x, y, z) \in P_{3} \mid m(y, x, x)=m(x, y, x)=m(x, x, y)\right\}
$$

для любых значений $x \in\left\{a_{1}, a_{2}, \ldots, a_{s}\right\}, y \in E_{k}$. Для любых $a_{1}, a_{2} \in E_{k}, a_{1} \neq a_{2}$ пусть

$$
d_{a_{1}, a_{2}}(x, y, z)=C_{k, a_{1}}^{2}\left(C_{k, a_{2}}^{2}(x, y), C_{k, a_{2}}^{2}(y, z), C_{k, a_{2}}^{2}(z, x)\right) .
$$

Легко видеть, что $d_{a_{1}, a_{2}} \in M Z_{3}\left(a_{1}, a_{2}\right)$. Предположим, что для любых $q$ различных значений $a_{1}, \ldots, a_{q}$ из $E_{k}, 2 \leqslant q \leqslant k-2$, класс $\left[\left\{C_{k+1}^{2}\right\}\right]_{G^{*}}$ содержит $d_{a_{1}, \ldots, a_{q}}(x, y, z) \in M Z_{3}\left(a_{1}, \ldots, a_{q}\right)$. Для любых $q+1$ различных значений $a_{1}, \ldots, a_{q}, a_{q+1}$ из $E_{k}$ пусть

$$
\begin{array}{r}
d_{a_{1}, \ldots a_{q+1}}(x, y, z)=C_{k, a_{q+1}}^{2}\left(C_{k, a_{1}}^{2}\left(d_{a_{1}, \ldots, a_{q}}(x, y, z), d_{a_{2}, \ldots, a_{q+1}}(x, y, z)\right),\right. \\
\left.C_{k, a_{2}}^{2}\left(d_{a_{1}, \ldots, a_{q}}(x, y, z), d_{a_{1}, a_{3}, \ldots, a_{q+1}}(x, y, z)\right)\right) .
\end{array}
$$

Легко проверить, что $d_{a_{1}, \ldots, a_{q+1}} \in M Z_{3}\left(a_{1}, \ldots, a_{q+1}\right)$. Тем самым доказано, что $\left[\left\{C_{k+1}^{2}\right\}\right]_{G^{*}}$ содержит мажоритарную функцию $d_{0, \ldots, k-1}(x, y, z)$. Согласно [9] в этом случае $\operatorname{ORD}[\{f\}]_{G^{*}} \leqslant k^{3}$. 
Пусть $f(x, \ldots, x) \neq x$ и $|\operatorname{Im} f(x, \ldots, x)|=k$.

При $k=3$, если $f \in L^{3}$, то согласно $[13] \operatorname{ORD}[\{f\}]_{G^{*}} \leqslant 2$, а если $f \in A_{3}^{0}$, то согласно [12] ORD $[\{f\}]_{G^{*}} \leqslant 3$.

Пусть $k=4$. При $f \in V_{4}^{4} L_{4}^{0} \backslash\left(S_{4}^{4} L_{4} \cup P V_{4}\right)$ нетрудно доказать соотношение $[\{f\}]_{G^{*}}=[\{x \dot{+} y \dot{+} z \dot{+} 1\}]_{G^{*}}=V_{4}^{4} L_{4}$. При $f \in V_{4}^{4} L_{4} \backslash\left(A_{4}^{4} L_{4} \cup V_{4}^{4} L_{4}^{0}\right)$ легко проверить, что $[\{f\}]_{G^{*}}=[\{3 x+2 y \dot{+} 1\}]_{G^{*}}=V_{4}^{4} L_{4}$. При $\left.f \in N L_{4} \backslash\left(P V_{4} \cup C E_{4} \cup V_{4}^{4} L_{4}^{0}\right)\right)$ нетрудно доказать, что $[\{f\}]_{G^{*}}=[\{x+y\}]_{G^{*}}=N L_{4}$. При $\left.f \in L L_{4} \backslash\left(N L_{4} \cup V_{4}^{4} L_{4} \cup L L_{4}^{1}\right)\right)$ согласно [6] $[\{f\}]_{G^{*}}=[\{2 x, x+y\}]_{G^{*}}=L L_{4}$. При $\left.f \in V Q L_{4} \backslash\left(V_{4}^{4} L_{4} \cup V Q L_{4}^{\alpha}\right)\right)$, аналогично подслучаю III. 2 получим, что

$$
[\{f\}]_{G^{*}}=\left[\left\{x^{2}+y^{2}+x+1\right\}\right]_{G^{*}}=V Q L_{4} .
$$

При $\left.f \in Q L_{4} \backslash\left(V Q L_{4} \cup L L_{4} \cup S Q L_{4}^{+}\right)\right)$согласно [6] $[\{f\}]_{G^{*}}=\left[\left\{x+y, x^{2}\right\}\right]_{G^{*}}=Q L_{4}$. При $f \in V_{4} \backslash\left(V_{4}^{\alpha} \cup V Q L_{4}\right)$ имеем $f(x, \ldots, x)=x \dot{+} c$ для некоторого $c \in E_{4}$ и $f\left(x_{1}, \ldots, x_{n}\right) \dot{+} c \in V_{4}^{\alpha}$. Отсюда аналогично подслучаю III.3 находим, что $\mathrm{ORD}[\{f\}]_{G^{*}}=\mathrm{ORD}[\{f \dot{+} c\}]_{G^{*}} \leqslant 4^{3}$. При $f \in P_{4} \backslash\left(V_{4} \cup Q L_{4} \cup P_{k}^{\alpha} \cup S L P_{k}\right)$ согласно $[6][\{f\}]_{G^{*}}=P_{4}$, что означает, что ORD $[\{f\}]_{G^{*}} \leqslant 2$.

Пусть $k \geqslant 5$. Тогда $f \in P_{k} \backslash\left(P_{k}^{\alpha} \cup S L P_{k}\right)$. Подстановкой переменных из $f$ можно получить $g(x) \in P_{k} \backslash P_{k}^{\alpha}$. Согласно теореме $1[\{f, g\}]_{G^{*}}=P_{k}$, т. е. ORD $[\{f\}]_{G^{*}} \leqslant 2$. Теорема доказана.

\section{Список литературы}

1. Яблонский С. В., Гаврилов Г. П., Кудрявцев В. Б. Фунжции аләебры логики и классы Поста. Наука, Москва, 1966.

2. Янов Ю. И., Мучник А. А. О существовании $k$-значных замкнутых классов, не имеющих конечного базиса. Докл. АН СССР (1959) 127, №1, 44-46.

3. Яблонский С. В. Функциональные построения в $k$-значной логике. Труды МИАН CCCP (1958) 51, 5-142.

4. Каргаполов М. И., Мерзляков Ю. И. Основы теории групп. Наука, Москва, 1982.

5. Мальцев А. И. Симметрические группоиды. Матем. сб. (1952) 31, №1, 136-151.

6. Нгуен Ван Хоа. О семействах замкнутых классов $k$-значной логики, сохраняемых всеми автоморфизмами. Дискретная математика (1993) 5, №4, 85-106.

7. Кудрявцев В. Б. Функциональяые системы. Издательство МГУ, Москва, 1982.

8. Мальцев А. И. Об одном усилении теорем Слупецкого и Яблонского. Алгебра и логика (1967) 6, №3, 61-74.

9. Марченков С. С. О замкнутых классах в $P_{k}$, содержащих однородные функции. Препринт Ин-та прикладной математики им. М. В. Келдыша АН СССР, 1981, №35.

10. Нгуен Ван Хоа. О структуре самодвойственных замкнутых классов трехзначной логики $P_{3}$. Дискретная математика (1992) 4, №4, 82-95.

11. Марченков С. С. Классификация алгебр со знакопеременной группой автоморфизмов. Матем. вопросы кибернетики (1989) 2, 100-122.

12. Марченков С. С., Деметрович Я., Ханнак Л. О замкнутых классах самодвойственных функций в $P_{3}$. Методы дискретного анализа в решении комбинаторных задач (1980) 34, 38-73. 
13. Baginszki J., Demetrovics J. The Structure of Linear Classes in Prime-Valued Logics. Discrete Math. Banach Center publication, Warsaw, 1982, 105-123.

14. Нгуен Ван Хоа. Описание замкнутых классов, сохраняемых всеми внутренними автоморфизмами $k$-значной логики. Докл. Академии наук Белоруссии (1994) 38, №3, $16-19$

Статья поступила 21.03.94. 